\title{
Wild Plants for Sale in the Markets of Pécs Then and Now (Baranya, Hungary)
}

\author{
Andrea Dénes \\ Janus Pannonius Museum, Department of Natural History
}

\begin{abstract}
Based on ethnographic and botanical sources as well as observations between 2012 and 2015, we have data about the sale of 130 species of wild plants in the markets of Hungary's fifth largest city. Most species, 98 of them, were sold as bouquets or wreaths, as ornamental plants. Sources reported sales of 67 wildflowers in the past, while between 2012-2015 they offered a total of 57 species in ornamental bouquets at the market but only 23 of the species sold in the past were among them. The main reason for changes in the wildflower species is that several species have become protected and hence their sale prohibited. Based on the available data, only 30 species of herbs and edible plants gathered in the wild were sold - 10 species as wild vegetables, 6 species as flowers, and 18 species as fruits. Today, of the edible wild species, mostly wild fruits and wild onions are available on a regular basis. Because the sale of medicinal plants became regulated very early on, their sale in the markets is not common, available mostly through wholesalers and already processed. Even today it is mostly edible herbs that are available in the market. During the 4 years of observation, they also sold 38 mushroom species in the markets of Pécs as forest products.
\end{abstract}

Keywords: wild food plant, wild fruit, wild vegetable, collection of medicinal plants, sale of wild plants, foraging, ethnobotany, open market, traditional ecological knowledge

\section{INTRODUCTION}

Wild plants collected in the wild are sold at the markets partly as cut flowers, bouquets of wildflowers, and partly as medicinal, herbal or edible plants. The use of nature's goods and the sale of wild plants in the market are not as common nowadays, but it seems to be reviving in Hungary. Considering a number of European countries (DoGAN et al. 2013; DogAN - NeDELCHEVA 2015; LuCZAJ et al. 2012, 2013), it can be established that it is traditionally in Mediterranean countries that the market sale of wild plants has endured, and, according to recent research, the use of wild plants is still significant in several regions of Transylvania (DÉNES et al. 2014; PAPP - HoRváTH 2013), and there is data about the market sale of numerous wild fruits (e.g., blueberries, wild strawberries) and wild vegetables (nettle, sorrel, docks, pilewort, coltsfoot, hop shoots) as well as wildflowers 
(e.g., dogtooth violet, liverwort, fritillaria) (BABAI ex litt. 2015; BARTHA 2015; DÉNES ined. 2015; SzÉKELY ex litt. 2012). Elsewhere in Europe the use of forest products and consequently their market availability have declined. The sale of mostly mushrooms, wild fruits, and a few wild vegetables have persisted in the twentieth century, but their rediscovery in the $21^{\text {th }}$ century can be observed in almost all countries. The situation is the same in Hungary - aside from forest mushrooms, only a very few species gathered in nature are being used today as they were used in the past, and data about their sale are few and sparse. The market supply of freshly foraged herbs has been suppressed in the last decades by readily accessible drugs and a good healthcare system, while the emerging demands were met through organized sales of processed herbal supplements. Wild plant foods - especially in a country with an increasing number of fertile lands due to the cultivation of lowlands and hill countries - have also been almost completely suppressed, their sale not very common. As ornamental plants, wildflowers appeared on the market only about a century ago, after World War I, and by the end of the century they were sold in bulk each spring. Due to environmental protection laws at the end of the $20^{\text {th }}$ century, their supply has declined, and by today the varieties of sold species have largely changed. 20 years after the regime change, I observed for 4 years the wild plant supply of the markets of a major Hungarian city surrounded by a diverse natural environment, rich in forests and grasslands, yet with markets that are some of the most expensive in national standards.

\section{MATERIALS AND METHODS}

For the study of today's practices of wild plant sales in Pécs, I frequented the markets of Pécs from 2012 to 2015, along with its farmers' markets and occasional fairs (e.g., Christmas market). I found the most wild plant sales in the market hall hosting the largest vegetable and flower market in Pécs, so I visited it on a daily basis in the more important periods of wild plant sales (spring, fall, holidays). During the four years, I spoke with 28 sellers, getting to know more and more about their gathering and selling practices, and I also asked three market supervisor about their prior observations. The longest-employed supervisor was able to look back on the last 20 years. Data of species sold that was observed by me at least once and/or mentioned in interviews and easily identifiable has been entered into the summary table (Table 1) as an unpublished source marked Dénes 2015 ined. Data of past wild plant sales in Pécs - especially for the period between 1940 and 1980 - was compiled from information found in botanical, ethnographic and environmental conservation literature, as well as in the ethnographic repository and photographic database of the Janus Pannonius Museum. The data from this period is sporadic, but despite this, we can learn a lot from the practices of the time. I supplemented my own observations with information from five colleagues from Pécs, all of whom have worked or are still working in environmental conservation and who were able to look back over the last 10-20 years. I received data from László Wágner (WÁGNER 2015 ex litt.), Sándor Völgyi (VöLGYI 2015 ex litt.), Balázs Kevey (KevEY 2015 ex litt.) Gábor Nagy (NAGY 2015 ex litt.), and Géza Vágner (VÁGNER 2015 ex verb.).

The study site is Pécs, Hungary's fifth largest city, a regional center and the seat of Baranya County. The population is over 145,000 today. The city that has existed since 
Roman times is situated in the southwestern foothills of the Mecsek Mountains, at the junction of three geographic regions: the Mecsek Mountains, the Baranya Hills, and the Dráva Plain. The proportion of natural habitats in the region is high. The city is bordered on the north by unbroken forested lands, and on the south by sometimes still swampy, flat areas. The flora of Pécs' surroundings is one of the richest of our domestic landscapes in terms of its absolute and relative number of species (WIRTH et al. 2010:71-72). The county is also home to Hungary's most forested lowland landscapes, the Dráva Plain. In the spring, nature awakens here first in the country, thus the very first willow catkins and wild garlic bouquets are available in the markets of Pécs. The ethnography and history of the markets and fairs of Pécs until the middle of the last century are described in a study by Imre Dankó (DANKó 1965). In the past, the marketplaces were the city's large public spaces, and Baranya's diverse, multi-ethnic population sold their goods here, but Pécs' weekly fairs may have had an even larger agglomeration. Today the city operates a market hall, and three smaller marketplaces, but several other private, occasional farmers' markets and craft fairs also operate to serve the demand. According to KIRÁLY (2011:89), Pécs' markets are mentioned among the most expensive domestic markets because the products almost always came from afar - in the past, mainly from producers on the Great Plain, currently also from abroad. For a long time, Bulgarian gardeners settled in the vicinity of Pécs at the end of the $19^{\text {th }}$ century also supplied the city with local vegetables, but today local producers are present on the market in very small proportions.

\section{HISTORICAL BACKGROUND OF WILD PLANT SALES}

\section{Wildflower sales in Hungary}

The sale of wildflowers as cut flowers appeared in towns during the difficult period after World War I. Primarily they sold early spring species because they were popular as the first signs of spring after a dark and cold winter in the city, and thus brought a modest income for the vendors. Well-known domestic botanists of the early $20^{\text {th }}$ century took stock of the wildflowers sold on the streets and markets of several big cities. PÉNZES (1926a, 1926b), RAPAICS (1932) and Boros (1924, 1947) all describe the condition and species of wildflower sales in Budapest. PÉNZEs (1926b) lists 89 species of wild plants in the markets of Pest. MÁTHÉ (1938) in Debrecen and TímÁR (1953) in Szeged both recorded the practices of wildflower sales in the respective cities' markets. Adolf Olivér Horvát, a botanist from Pécs - having had recorded sales of 61 species gathered in the wild - published a study in 1940 and again in 1942 about the wildflowers sold in the markets of Pécs. According to the studies of VIGA (1986:69-70), for example, the women of Cserépfalu in the Bükk mountains were "making a living" by gathering forest products all year round, and they would even go pick snowdrops near Pécs:

They started their gathering "in the early spring with the snowdrop, then came the violets, mushrooms, lily of the valley, mushrooms again, then raspberries, herbs (Christmas rose, belladonna, chamomile, centaury, black locust flowers, agrimony). The herbs were purchased by the village trader - they themselves did not really use them. But the rest they took to the market themselves. The gathering of snowdrops became fashionable in particular after World 
War II, although already quite a few people did it before the war (...) they pick snowdrops especially for Women's Day and Mother's Day, collecting them in the surrounding woodlands of the Bükk mountains, but they would also go to pick flowers in the remote Transdanubian forests (Pécs, Villány, Kaposvár, Dombóvár, Nagykanizsa), then bring the "harvest" to be sold in the markets of the capital, sometimes Eger and Miskolc."

I myself saw the process of making bouquets out of snowdrops gathered in bulk and piled high on tables pushed together in the waiting room of the Villány railway station near Pécs in the '90s. Snowdrops were popular not only in Hungary but also in the neighboring (then) Czechoslovakia, with bouquets delivered even by air to large cities (Új Szó 1973). The weekly magazine Hét, referring to Dunacsúny, wrote in 1976 that there were "some people who even bought cars from [the proceeds of] snowdrops" in the village dubbed "Hóvirágfalva" (Snowdrop Village), but the people living there did not mind because "no other village in the country has such a beautiful name" (SCHNEIDER 1976).

Initially botanists were less worried about the mass gathering of wild plants, but later they became more and more worried (ARADI et al. 1975:35; BOROs 1968; MOLNÁR V. 2014:41-45; NÉMETH - SEREgÉLYes 1982). The demand for wildflowers increased, especially for those blooming in early spring before the cultivated species, and became more than just a supplement to meager incomes, and thus more people got involved in the wildflower trade. Several species were declared protected by a 1982 act, the first of its kind in Hungary, in order to prevent further environmental damage caused exactly by wildflower gathering. ${ }^{1}$ Despite their protected status, some protected species still appeared on the stalls. I've also regularly seen colorfully painted feather grass (Stipa) sold at Baranya's famous pilgrimage site, Máriagyüd in the 1990s, and in those years the more common, protected flowers of the Mecsek also appeared in the markets of Pécs. For some species, their sales increased to worrisome degrees, so in $2001 \mathrm{WWF}$ Hungary launched a campaign called "Let the wildflowers live". In their brochure, they analyzed the contemporary situation, and they concluded that several protected species of plants were being sold in the metropolitan markets, and of the non-protected ones, the snowdrop was being threatened by the tremendous demand for it on International Women's Day (March 8), when most men gift their female friends, female relatives with a bouquet of snowdrops. On this day, and a few days before, many millions of snowdrops were put on the markets. As calculated by the conservation organization, just from Nagykanizsa, arriving by train, nearly 73 million snowdrops made it to the markets in the capital (KEREKI 2001). Snowdrops were eventually declared protected in Hungary in 2005. In those years, rangers often checked the markets and flower shops. They informed, warned, posted posters, and market supervisors did not allow the sale of snowdrops and other protected species, so now we can say that protected species are very rare in the markets, and certainly not in large quantities.

${ }^{1}$ 1/1982. (III.15.) OKTH Regulation - Hungarian Bulletin 1982, No. 14. 


\section{Historical overview of the market sales of edible wild plants}

Researchers of foraging economies considered the use of wild plants in Hungary to be most significant until the early $20^{\text {th }}$ century. Nature foraging was better preserved in landscapes that were less suitable for agricultural production but richer in natural habitats and forests, or even in rocky and arid mountainous landscapes (GUNDA 2001, ÚJvÁRI 1957). According to ethnographic and ethnobotanical sources, one could find some 216 wild plant species as food or herb on the tables of Hungarians living in the Carpathian basin (DÉNES et al. 2012:383). In the opinion of VIGA (1990:89),

\footnotetext{
“...foraging basically serves self-sufficiency, and foraged forest products become a commodity in larger quantities primarily with the development of the social division of labor. Their real customers are city/town markets, possibly nearby micro-regions that lack certain typical fruit or plant species due to different geographical conditions. However, these materials rarely serve as basic provisions, but rather appear in the markets of cities or other regions as a delicacy, a 'specialty', and it is exactly their 'otherness' that gives them value."
}

Of the forest foods, it was mainly wild fruits, juniper, pine and cumin seeds, but mostly mushrooms that made it to the markets from the regions where foraging was "an inalienable part of traditional culture" (VIGA 1990:91). The earliest data is about the market sales of the water chestnut (Trapa natans) occurring in the swamps along the great rivers. In 1902, Lajos Zoltay listed among the occupations in Debrecen from 200 years ago the "seller of water chestnuts", of which at that time there was only one at the market in Debrecen (ZoltaY 1902:23). Chervil (Chaerophyllum bulbosum) and corn salad (Valerianella) were sold in eighteenth-century Debrecen markets, or at least at the time this was recorded by student poets (ORTUTAY 1977). Also known are early sales of the ground-ivy (Glechoma hederacea) in the markets of Pest (VeSZELSZKI 1798:151). There is also mention of the sale of the sap of the birch (Betula pendula), birch water, in the 1700s; Ferenc Rákóczi, in a letter from 1760, mentions birch water, which "is not more expensive (...) than the Tokaj harvest" (Kiss 1929:4). Birch water was brought from the Nyírség to the Great Hungarian Plain, too, by itinerant vendors (Kiss 1929:4), and water chestnut was also sold along the Tisza, Danube, and Dráva (ANDrásfalvy 1965:16; Gunda 1956:23; NaGy 1917:839; Petrikovits 1943), and in Baja even in 1980, when Ortutay (1981) documented in a striking photo the water chestnut-selling women of Baja. Along the Dráva, in Baranya, elders still remember the vendors of water chestnuts from Cún, whose products sold out of carts they themselves have tasted as children (DÉNES ined 2015).

\section{RESULTS}

\section{The wildflowers of the flower market of Pécs in the past and today}

In light of available data, so far a total of 98 wildflower species (including shrubs and trees) have been sold as ornamental plants in the markets of Pécs (Table 1). The most complete record of sales has been published by Adolf Olivér Horvát (1942:60-61), 
listing 61 species, including several that are now protected. The lady orchid (Orchis purpurea), the pheasant's eye (Adonis vernalis), the spiny and spineless butcher's-broom (Ruscus aculeatus, Ruscus hypoglossum), feather grass species (Stipa sp.), and the since extinct cottongrass (Eriophorum sp.) have all turned up at the market. There is little data available from the next period. From the 1980s to 2005, sales of 19 species is known with certainty. They sold, among others, snowdrop (Galanthus nivalis), lily of the valley, species of mistletoe (Viscum album, Loranthus europaeus), catkin (Salix caprea), and rosehip (Rosa canina) bouquets, but the protected dogtooth violet (Erythronium dens-canis), butcher's-broom (Ruscus aculeatus), leopard's bane (Doronicum spp.), and in small bouquets even the primrose (Primula vulgaris) made an appearance in the market. By now the species sold have been to a large extent replaced. In the last four years, 57 species have been tied into bouquets in the market, but only 23 of the species were the same as those sold a long time ago. Still sought after today are bouquets of violet (Viola spp.) and lily of the valley (Convallaria majalis) (Figure 1), and sometimes meadow sage (Salvia pratensis), woodruff (Asperula odorata) (Figure 2), or oxeye daisy (Leucanthemum vulgare) also get mixed into bouquets of garden flowers. Seemingly late discoveries, of the summer wildflowers the hoary cress (Lepidium draba) and cypress spurge (Euphorbia cyparissias) (Figure 1), both easily gathered from roadsides, are sold by many, and they have been incorporated into Biedermeier bouquets (Figure 3) supplemented by vividly colored garden flowers. Many other common species also appear on the market, just-blooming or colorful species, shrubs and trees, too, such as black locust (Robinia pseudoacacia), wild privet (Ligustrum vulgare), spindle berries (Euonymus spp.), Chinese lantern (Physalis alkekengi), and bladder campion (Silene vulgaris). The leaves of ivy (Hedera helix) and horse chestnut (Aesculus hippocastanum), and many grasses (Anthoxanthum odoratum, Briza media, Arrhenatherum elatius) are only supplemental "greens" in the bouquets. Many are selling the green shoots of the Scotch broom (Cytisus scoparius) as "horsetail" (Figure 4). At Easter the catkins of the pussy willow (Salix caprea) (Figure 10), before Christmas the yellow mistletoe (Loranthus europaeus) and white mistletoe (Viscum album) are an inevitable mass commodity (Figure 4). Bouquets of hawthorn (Cratageus monogyna) and rosehip (Rosa canina) wrapped with ivy or pine boughs (Figures 4-5) have long been popular at this time - there are people who have been selling them for 20 years. Today, as in the past, part of the winter supply is the colorfully painted cutleaf teasel (Dipsacus laciniatus) (Figure 6) and bushgrass (Calamagrostis epigeios), but even the milkweed's (Asclepias syriaca) dry "parrot beaks" may be incorporated into bouquets nowadays (Table 1). 


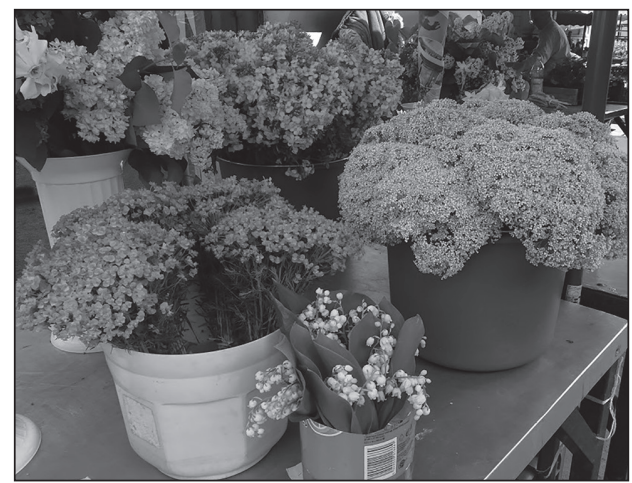

Figure 1. Euphorbia cyparissias, Lepidium draba and Convallaria majalis on the market. Pécs, Hungary, 2015. (Photo by Andrea Dénes)

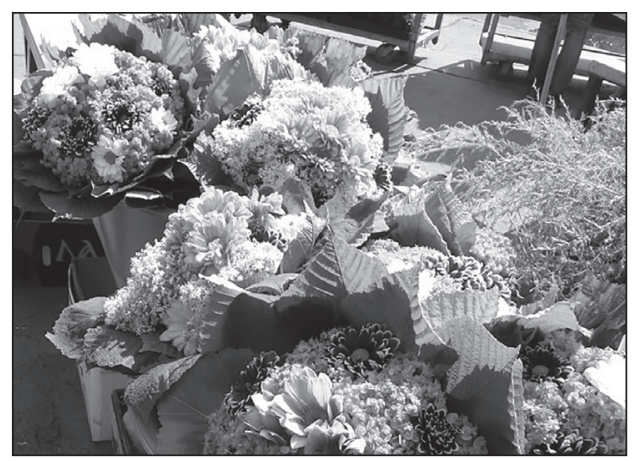

Figure 3. Hoary cress and cypress spurge sold by many, and they have been incorporated into Biedermeier bouquets. Pécs, Hungary, 2015. (Photo by Andrea Dénes)

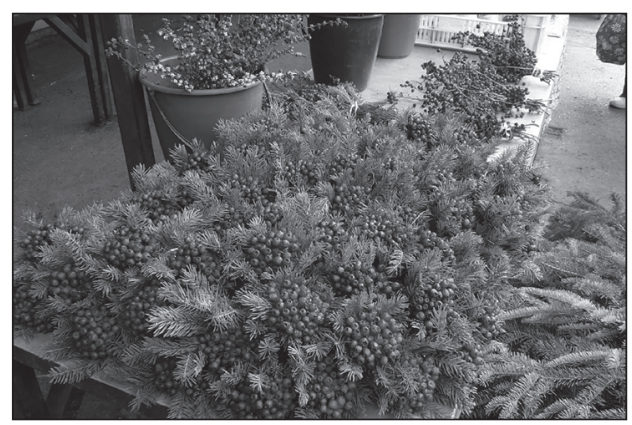

Figure 5. Bouquets of hawthorn (Crataegus monogyna) wrapped with pine boughs. Pécs, Hungary, 2015. (Photo by Andrea Dénes)

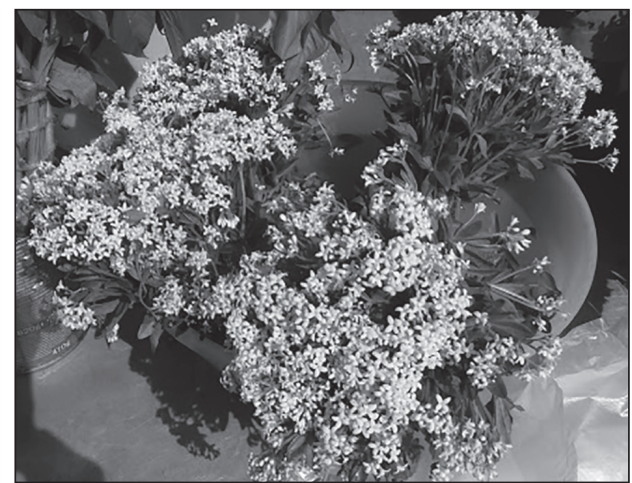

Figure 2. Selling of Asperula odorata bouquets as ornamental plants. Pécs, Hungary, 2015. (Photo by Andrea Dénes)

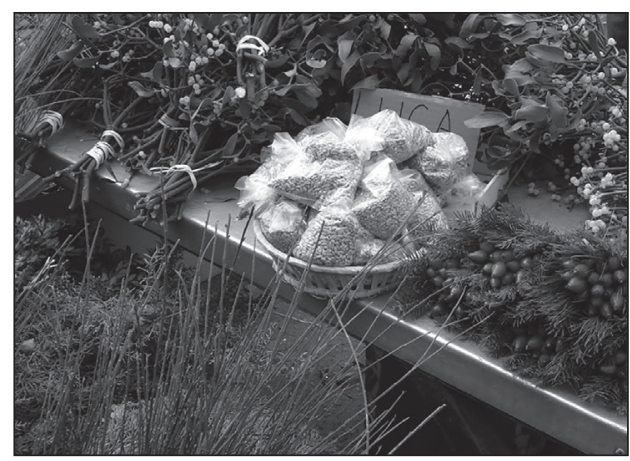

Figure 4. Cytisus scoparius, Rosa canina, Loranthus europaeus and Viscum album on the Chrsitmas market. Pécs, Hungary, 2015. (Photo by Andrea Dénes)

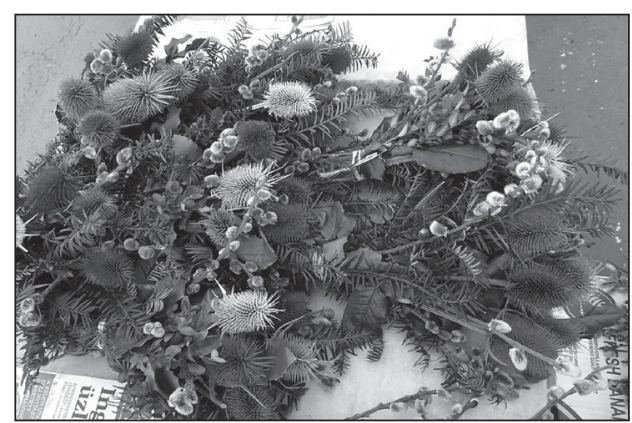

Figure 6. Colorfully painted cutleaf teasel (Dipsacus laciniatus) and very first willow catkins (Salix caprea) at the end of winter on the market. Pécs, Hungary, 2015. (Photo by Andrea Dénes) 


\section{The wild food plants of the markets of Pécs}

In Baranya County, sources have recorded 47 wild plant species used as food (Borsos et al. 76:4; FÜVESY 1997; GUNDA 1956:23; KIss 1980:20-21; Z. KISS 1994:178-179; MÜLLER 1973; NAGY 1942:269-273; NYILASSY 1951; VÁRÓCZI 2011; ZENTAI 1966:185-186). Data about sales of far fewer species have survived. Based on all available data, a total of 30 edible wild plants turned up in Pécs markets. 10 species of plants were sold as vegetable (green leaf, young shoot), 6 species were offered for their flower, and 18 species collected in the wild were available as fruit or seed. Of the wild fruits, it is especially blackberry (Rubus fruticosus), rosehip (Rosa canina), and blackthorn (Prunus spinosa) sales that appear to be continuing to the present day. Based on the data from the ethnographic interviews from the 1950s in Zengővárkony (NYILASSY 1951:15, 47-48), of the forest fruits, the wild strawberry (Fragaria spp.) was most often gathered and taken to the market. A then 82-year-old informant said that even earlier, in the time of her mother, they sold wild strawberries in markets. At ripening time, they set out for the woods at 2 in the morning and picked them sometimes until 4 in the afternoon. The next day they took them to the local or to the Pécs market (NYILASSY 1951:48). Nowadays wild strawberries can be found only rarely, as a delicacy, picked with the stem, bound in small bouquets. On the other hand, rosehips and the jam made from them, the hecsedli, have been in demand throughout the times. There is demand for the wild blackberry (Rubus fruticosus) today, and it has been a long-time favorite, at least according to Kovács Jánosné, a former collector: "the blackberry is a very valuable fruit, they pay for it well" (NyILASSY 1951:47). Of the wild fruits, in addition to the above, the Cornelian cherry (Cornus mas) and the hawthorn (Crataegus monogyna) are sought after, but there is no data about their former sales (Figures 7-9).

Of the 10 wild vegetables sold, the wild garlic (Allium ursinum) is the most common in the markets of Pécs (Figures 8-9). The nationally soaring career of this species over the past decade began in the vicinity of Pécs. The first Orfü Wild Garlic Festival, held in 2004, is considered to have caused the increased interest in wild garlic (BARINA 2014; MÉsź́ros B. 2015), so much so that due to the plant now being gathered "by the tons" (FEHÉR - VARGA 2013), conservationists are considering having the wild garlic declared protected (BARINA 2014; TEMESI 2014). There are no traces of its sale in the past, and not a lot of data in the county about its consumption. What is certain is that the people of Nagyváty gathered and consumed it (NAGY 1942:270, 307). Nonetheless, wild garlic is common in the natural forests of Baranya County. Since about 10 years ago, its sale has often started in early February and lasted up until early June. In the four years of observation, of the relatively thin leafed wild onions, two species (Allium scorodoprasum and $A$. vineale) were often available in the winter and early spring as "wild chives" and "wild garlic" (Figures 8-10). Their barren, winter-spring leaves were regularly sold by two vendors. Pilewort (Ranunculus ficaria) was rarely available (Figure 9), but there is a demand for its restaurant delivery (VöRös ex verb). I have only seen hop shoots (Humulus lupulus) in the market once, purslane (Portulaca oleracea) was supplied by order by a vegetable vendor. Chickweed (Stellaria media), dandelion (Taraxacum officinale) and fresh nettles (Urtica dioica) were sold alone, and mixed with garden salad leaves. 


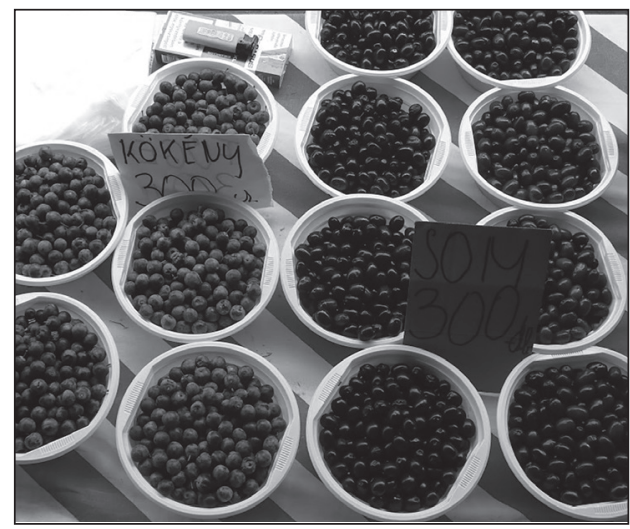

Figure 7. Cornelian cherry (Cornus mas) and the blackthorn (Prunus spinosa) are sought after, but there is no data about their former sales. Pécs, Hungary, 2015. (Photo by Andrea Dénes)

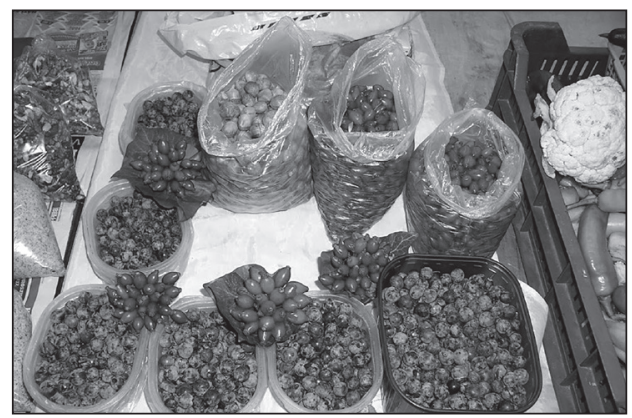

Figure 9. Blackthorn, rosehip and Turkish hazel on the market. Pécs, Hungary, 2012. (Photo by Andrea Dénes)

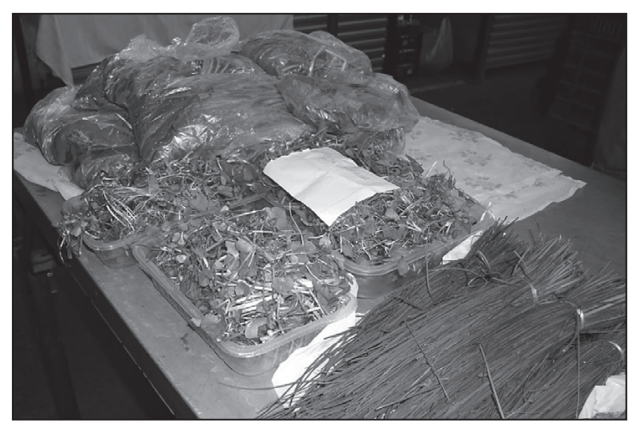

Figure 11. Selling Alllium ursinum, Allium scorodoprasum and Ranunculus ficaria as wild vegetable on the market in spring. Pécs, Hungary, 2012. (Photo by Andrea Dénes)

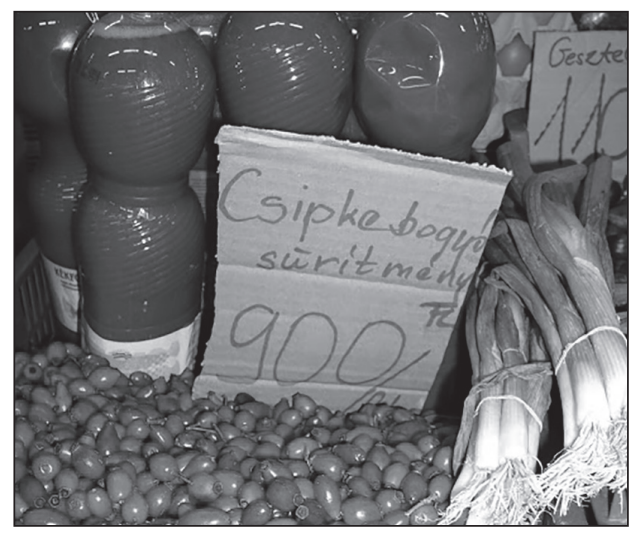

Figure 8. Fresh rosehip (Rosa canina) fruit and semifinished rosehip concentrate were sold every year on the market. Pécs, Hungary, 2012. (Photo by Andrea Dénes)

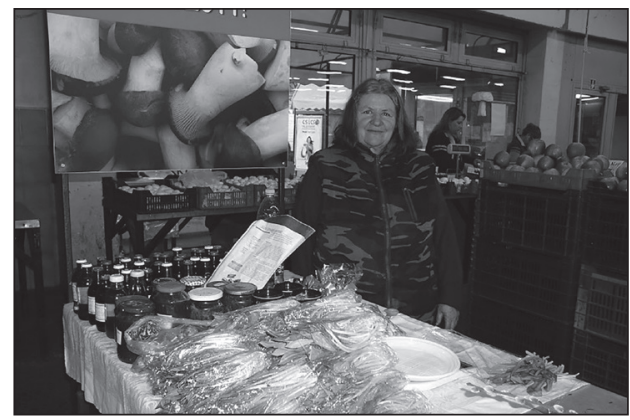

Figure 10. Marika is a reliable source for many species of edible wild plants at the market. Pécs, Hungary, 2012. (Photo by Andrea Dénes)

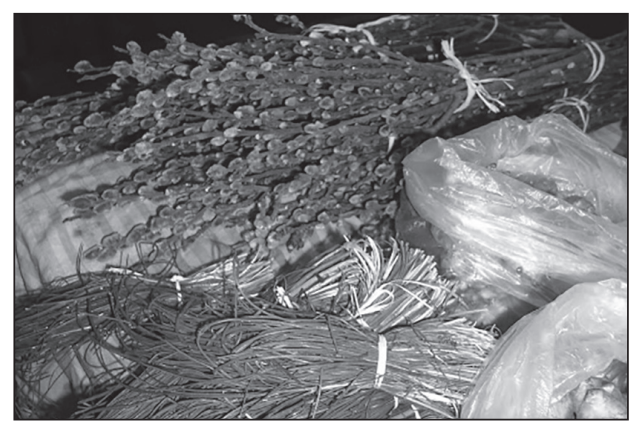

Figure 12. Willow catkins (Salix caprea) and wild garlic (Allium scorodoprasum) bouquets are available in the markets of Pécs. Pécs, Hungary, 2015. (Photo by Andrea Dénes) 


\section{Herbs for sale}

Foraged herbs were less likely to make it to urban markets in the past, and today it is still very rare, mostly because selling fresh or dried herbs in bulk or in bunches is prohibited by relevant rules. In the past, they collected them to satisfy the needs of the family, but there were some who bought them from itinerant vendors or from local herbalists: "We never pick the teas, the Gypsies bring them" (Widow Szücs Józsefné, in NyILASSY 1951:54). Only the best-known, time-tested herbs were sought after in the Pécs markets: "Chamomile, linden I collect, but I will not sell it, it is not valuable (...) I'll also pick centaury, and sell it too (...) in the market, in clumps like the 'Mecsek tea' (Melittis melissophyllum). But it is not as expensive, because only those buy it that have stomach aches and are convinced that it is good. And the 'Mecsek tea' (Melittis melissophyllum) is not just medicine" (Kovács Józsefné in NYILASSY 1951:57). In Hungary, the organized acquisition, processing, and trade of herbs (TÉTÉNYI 1995:504-505) began very early, in 1904, with the foundation of the Research Institute of Medicinal Plants in Cluj, which still operates today. There are few data about market sales of herbs, such as Tamás Grynaeus', which commemorates the last herbalist woman in the Szeged market (1964). Today the herbalist man of the Pécs market sells packaged, labeled herbs, tinctures and ointments in compliance with the law (as I saw in 2012-13, he now has an independent shop near the market). In the case of herb sales in Pécs markets and fairs, we have data about 10 species (Table 1). The 'Mecsek tea' or bastard balm (Melittis melissophyllum) used to be very sought-after, was repeatedly brought to market, purchased by Pécs pharmacies, as well as bought by the quintals by consumers (NYILASSY 1951:6-7, 30, 50-55). It is no longer sold. Linden flower also used to be collected; ethnographer László Mándoki documented its sale in 1970 at the Pécs market with a photo (Figure 13). Today, it is rarely for sale at the market. Similarly, only once in a while did dried nettles, ribwort plantains, oregano, thyme or the wild mint appear among the market goods. Those who might order them can find a vendor who collects and delivers them on demand. Of the flowers of wild plants, one could occasionally get fresh elderflowers, black locust flowers, and dried mallow flowers (Figure 14). Jam made from dwarf elderberries (Sambucus ebulus) was also sold (Figure 15), but the most common for tea and jam alike were fresh haw and rosehips (Figure 16) (Table 1).

\section{Mushrooms for sale}

This study focuses on the sale of wild plants, but mushrooms played an important role at all times in forest foraging and in the market sale of forest products. The market sale of mushrooms has been a longstanding and safely continuing activity primarily in the cities of Transdanubia and the central mountainous regions, of which we have data from Pécs (Figure 17). In the Pécs market, they mainly sold the more frequent mushrooms known and sought by the customers, whereas today only those are sold that are allowed by Hungarian law. ${ }^{2}$ Many of the wild plant vendors also collect and sell mushrooms. It

\footnotetext{
${ }^{2}$ 107/2011. (XI. 10.) VM ordinance
} 
was so in the past, too. The "Mushroom King" of Zengővárkony - as he was called in the village - foraged for and sold mushrooms, herbs and wild fruits. Started by necessity, he had been doing it for 22-23 years by the time of the 1951 interview, and made a good living with it. "I have a knack for mushrooming like for a profession, though it is very burdensome" (NyILASSY 1951:1-2, 10-17). Today, sales of mushrooms and the necessary inspections and monitoring can only be resolved in the Pécs market hall. In the four years of observation, they sold 38 species of mushrooms. Porcini (Boletus) species are in highest demand, along with chanterelle (Cantharellus cibarius) and Scotch bonnets (Marasmius oreades). The mushrooms arrive to the Pécs mushroom markets mainly from Baranya County and the neighboring Somogy County, but local mushroom vendors also ship to the city markets of the Great Plain that is less rich in fungi; I have encountered mushroom merchants from Pécs at the markets of Szeged and Baja as well.

\section{Wild plant sales practices}

Wild plant sales in Pécs are seasonal, but the season adapts to customer needs beyond the collection period, especially in the case of wildflowers. In periods of greater demand there are more vendors, and vendors normally selling other goods will supplement their goods with popular seasonal species, most often wild garlic. According to approved license applications at the Pécs market hall, forest products (wild plants and mushrooms) were sold by 55 vendors in 2015. Among the edible wild plants, fresh wild garlic and wild fruits are sold most. Several wild vegetables were only sold by two vendors over the four years. Of the 28 interviewees, six were selling more or less continuously - four flower and two vegetable vendors. Their stocks almost always included wild plants, sometimes they did not even sell anything else. 13 people were selling wild mushrooms or plants only seasonally, but never garden produce: "in the spring wild garlic (...) when I run out of mushrooms in the fall, I go mistletoe picking". Occasional vendors (nine people) sold the most sought-after wild garlic and wild fruits, or else Christmas and wildflower ornamental bouquets. The foraging site is Baranya County. Most of the collectors/sellers are not from Pécs, but commute from a nearby settlement. Some bring their goods themselves by bus: “(...) write this, honey, that old granny brings two bags, and takes back two bags of what she doesn't sell'. Several have been selling wild plants for a long time, 15, 20, even 25 years. Most of them mentioned rosehips as the longest-collected plant. The supply of wild plants also showed differences from year to year during the four-year observation, thus a trend could not be determined as it was dictated primarily by the amount of crops. In 2015, for example, the Cornelian cherry grew very well, many were selling it, whereas in previous years it was not at all on the market. In 2014, no hawthorn or blackthorn grew in the area, thus there was not a large supply of these on the market, while in 2013 more than 15 vendors were selling them. And in 2013 the just-sprouted wild garlic was permanently covered with snow, thus the sales started very late. 


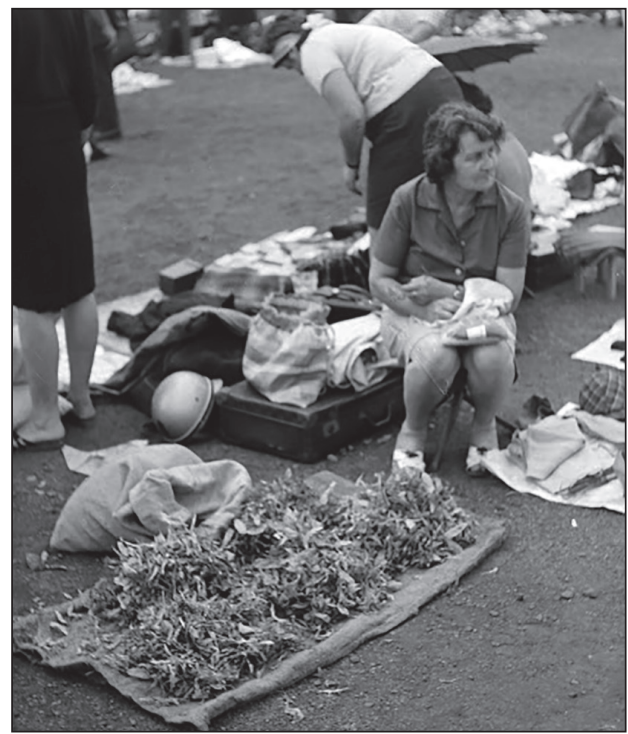

Figure 13. Selling of lindenflowers on the marketplace. Hungary, Pécs, Photo: László Mándoki. Photo Archive of Janus Pannonius Museum / Lsz. 19978; 1970.

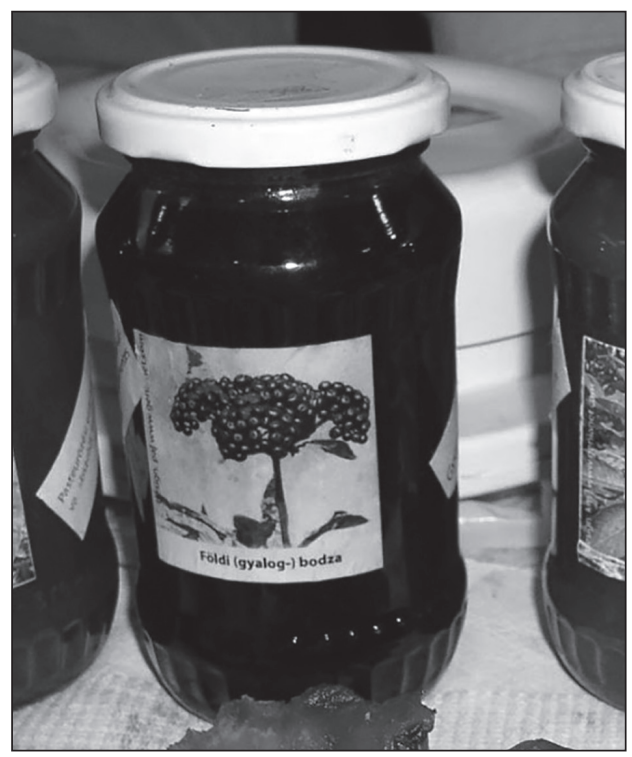

Figure 15. Selling dwarf elder (Sambucus ebulus) marmelade on the market. Pécs, Hungary, 2012. (Photo by Andrea Dénes)

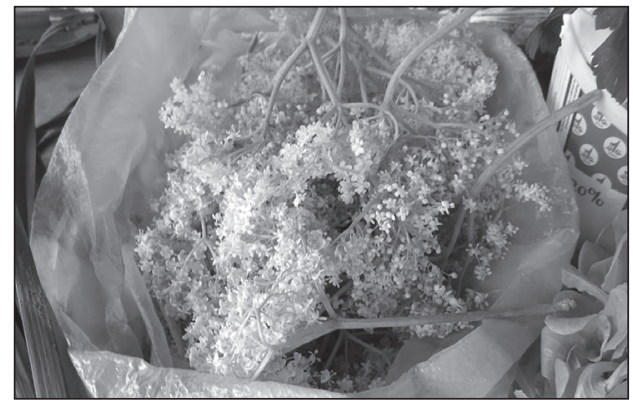

Figure 14. Selling of Sambucus nigra flowers for syrup and for tea. Pécs, Hungary, 2012. (Photo by Andrea Dénes)

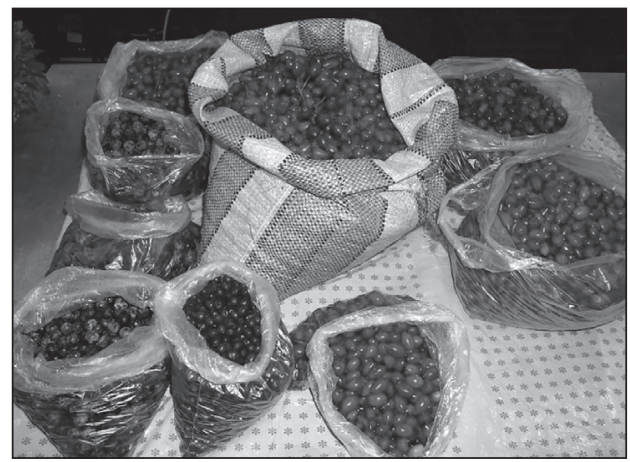

Figure 16. Hawthorn, blackthorn and rosehip on the market. Pécs, Hungary, 2012. (Photo by Andrea Dénes)

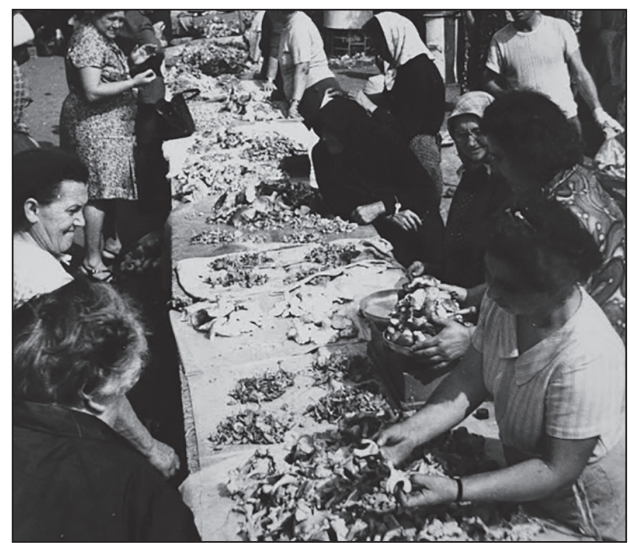

Figure 17. Selling of fresh, wild mushrooms in the 1970s on market of Irányi Dániel square. Hungary. Pécs, Photo Archive of Janus Pannonius Museum / Lsz. F1608. 


\section{DISCUSSION}

Wild plant sales are clearly present today in the markets of Pécs and have been in previous periods, too, but mostly only edible wild fruits and wildflowers sold as ornamental plants were offered. In Hungary today, there is very sparse data about the market climate of other cities, so there is no possibility for comparison, but it is surmised that Pécs combines a number of conditions that is favorable for the market accessibility of forest products. Its natural environment rich in forests is suited for foraging; there is a big enough demand for legal mushroom sales in its largest market, thus many people turned to forest product foraging and sell other species outside of mushroom season. There is demand for their commodities because wild plants, aside from being a specialty, can also be offered cheaper than the largely non-local and therefore more expensive flowers and vegetables.

Wild plant collecting - as well as selling - has long had and still has its real experts, specialists. One such specialist was the "Mushroom King" of Zengővárkony who collected wild fruits and herbs for decades (NYILASSY 1951:1-2,10-17), or Aunt Eta, a vendor in the Pécs market who is the "last woman to collect flowers and fruits in Bisse in the foothills of Tenkes" (SzABó 2012). Today it is Irénke who makes the most inventive bouquets of wildflowers and stems, creating decorative table ornaments and garlands out of seemingly nothing, or a decorated "Christmas tree" out of the long branchy shoots of the Scotch broom. Marika is a reliable source for many species of edible wild plants at the market (Figure 10). Wild onions, pilewort, elderflowers and others can be obtained from her both fresh and processed, conserved. Purslane can be obtained from Miklós, who brings it from his chemical-free garden. There are some who specialize in rosehips, following family tradition, grandparents who practiced rosehip-collecting and processing for 40 years. Although the majority of the vendors selling wild plants and mushrooms, in part or in full, exercises this activity with a re-learned knowledge, they almost always possess a certain family- or community-related sensibility rooted in the past for knowing wild plants and mushrooms and for forest foraging. Often it was the grandparents, the parents, or other relatives that collected, used, and occasionally sold wild plants and mushrooms. The old Gypsy man who sells wild onions, pilewort, and mushrooms and says he himself likes to consume these and gives advice about their cooking probably does not get his information about these species from the Internet. But nowadays gatherers and vendors probably glean and learn from each other, too. If a species is selling well, next time more vendors will offer them. To many of them, the sales proceeds are an important and necessary supplement or primary income. In Zengővárkony in the 1950s, there were "those who lived off of the forest" (NYILASSY 1951:9), and there are still some today.

From the market supervisors' and customers' perspective, the sale and purchase of edible wild species happens almost completely on a confidential basis. Looking back over the last 20 years, market supervisors remember seeing, aside from mushrooms, only rosehips (Rosa canina), mulberries (Morus) and blackberries (Rubus) in the market, as the wild garlic (Allium ursinum) only appeared about ten years ago. But while they are qualified to inspect all forest mushrooms brought to the market, they have not been trained to recognize wild plants, so they do not inspect, for example, the bunches of wild garlic to make sure no other similar and potentially poisonous wild plant got mixed in. Although they have good intentions, what they do not recognize and do not know for 
sure is edible, they cannot allow to be sold. They fear that in the case of the most soughtafter wild plants in the market, the hope of increased revenue may result in less care, coupled with a less attentive gathering.

\section{ACKNOWLEDGMENTS}

I would like to thank Babai Dániel, Gyenei Gábor, Handler Gábor, Kárpáti Ferenc, Kevey Balázs, Nagy Gábor, Németh Ibolya, Orbán Imre, Székely Annamária, Vágner Géza, Völgyi Sándor, Vörös Kálmán, and Wágner László for providing data, and Kőszegi Gábor, Burján István, and Sárközi Katalin for their help with ethnographic resources. 
Wild Plants for Sale in the Markets of Pécs Then and Now (Baranya, Hungary) 353

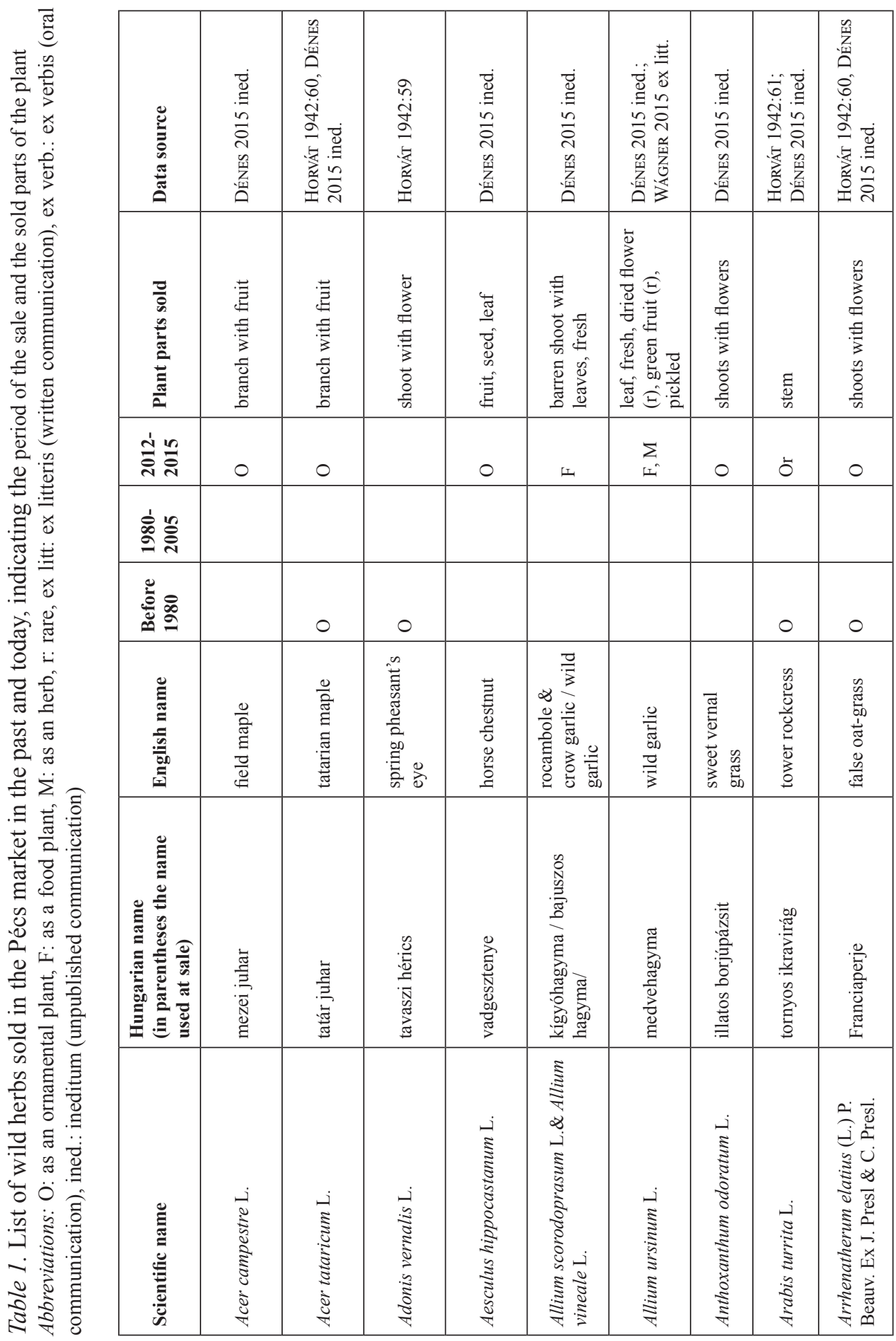




\begin{tabular}{|c|c|c|c|c|c|c|c|c|c|c|}
\hline 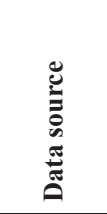 & 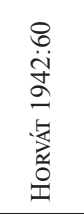 & 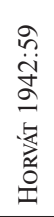 & 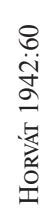 & 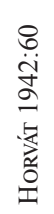 & 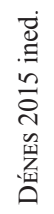 & 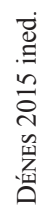 & 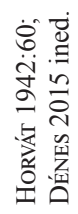 & 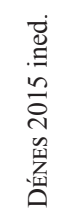 & 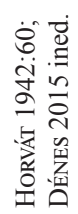 & 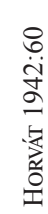 \\
\hline 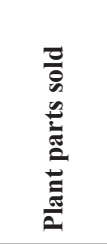 & 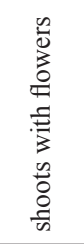 & 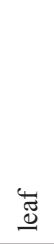 & 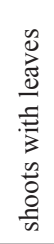 & 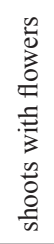 & 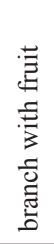 & 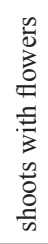 & 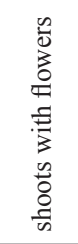 & 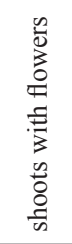 & 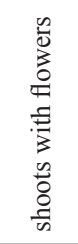 & 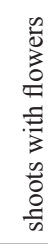 \\
\hline 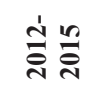 & & & & & $\ddot{\sigma}$ & 0 & 0 & 0 & 0 & \\
\hline ฆั่ & & & & & & & & & & \\
\hline 离 ま & 0 & 0 & 0 & 0 & & & 0 & & 0 & 0 \\
\hline 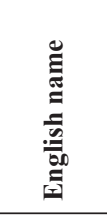 & 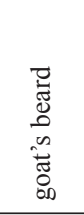 & 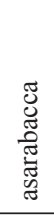 & 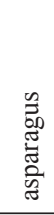 & 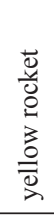 & 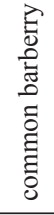 & $\frac{\mathbb{N}}{8}$ & 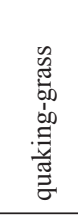 & 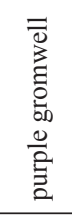 & $\begin{array}{l}\tilde{w} \\
\tilde{z} \\
\tilde{5} \\
\overline{5} \\
\bar{z} \\
\Xi\end{array}$ & 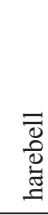 \\
\hline 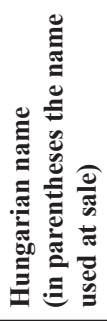 & 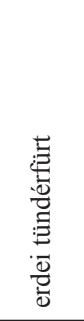 & 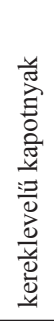 & 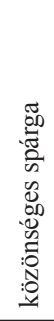 & 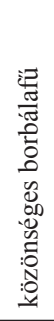 & 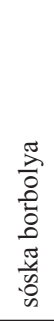 & 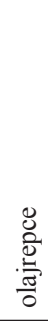 & 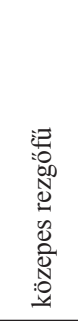 & 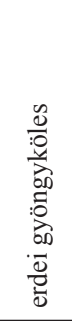 & 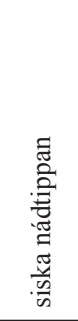 & 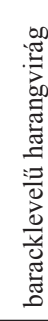 \\
\hline 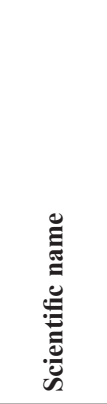 & 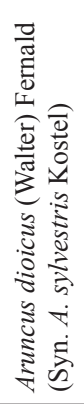 & 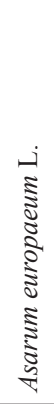 & 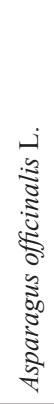 & 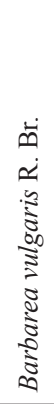 & 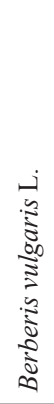 & 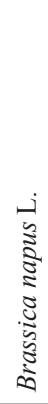 & 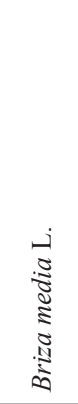 & 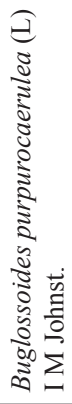 & 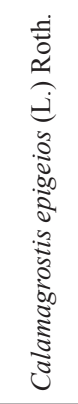 & 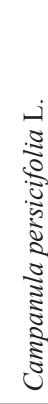 \\
\hline
\end{tabular}




\begin{tabular}{|c|c|c|c|c|c|c|c|c|c|c|}
\hline 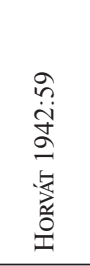 & 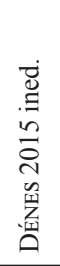 & 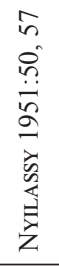 & 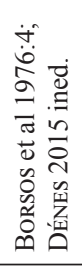 & 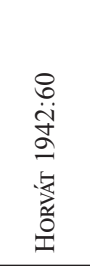 & 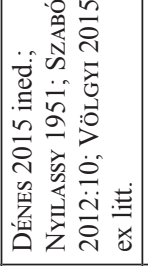 & 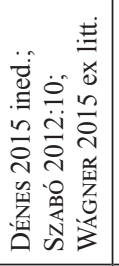 & 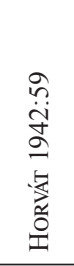 & 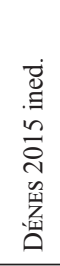 & 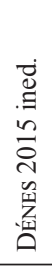 & 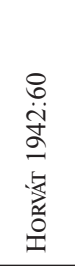 \\
\hline 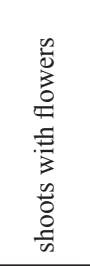 & $\underset{\mathscr{J}}{\mathscr{J}}$ & 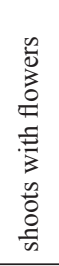 & 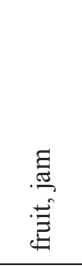 & 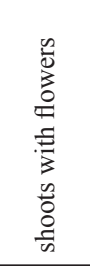 & 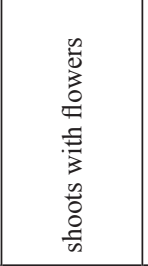 & 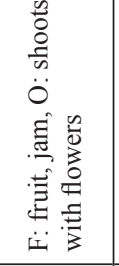 & 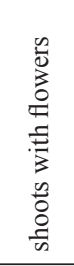 & 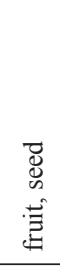 & 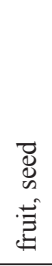 & $\begin{array}{l}n \\
0 \\
0 \\
0 \\
0 \\
0 \\
0 \\
0 \\
0 \\
0 \\
0 \\
0 \\
\frac{1}{n} \\
\end{array}$ \\
\hline & $0^{\circ}$ & & I & & 0 & ö & & 工 & 工 & \\
\hline & & & & & 0 & L. & & & & \\
\hline 0 & & $\Sigma$ & I & 0 & 0 & & 0 & & & 0 \\
\hline 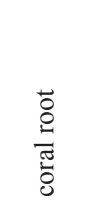 & 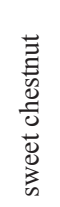 & 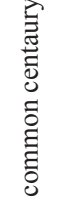 & 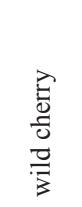 & 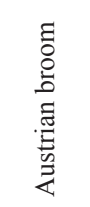 & 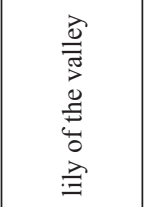 & 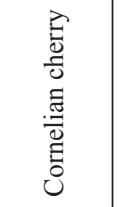 & 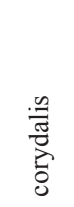 & $\begin{array}{l}\overline{\mathscr{N}} \\
\text { }\end{array}$ & 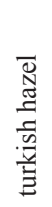 & 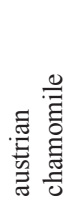 \\
\hline 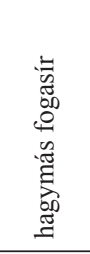 & 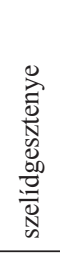 & 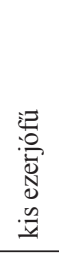 & 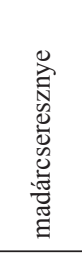 & 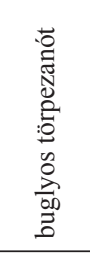 & 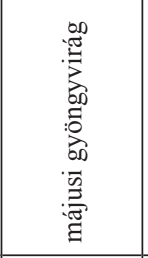 & $\begin{array}{l}\text { ह } \\
0 \\
0 \\
0 \\
00 \\
0\end{array}$ & 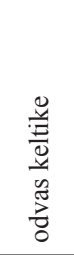 & 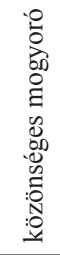 & 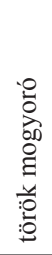 & 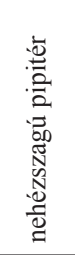 \\
\hline 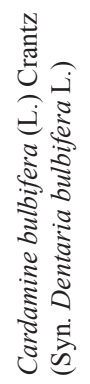 & 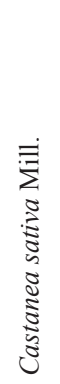 & 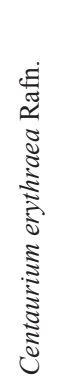 & 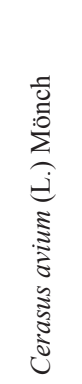 & 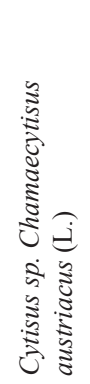 & 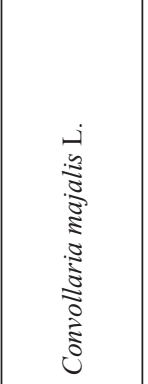 & 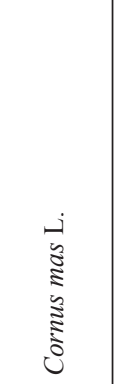 & 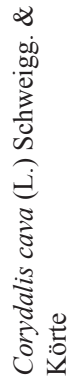 & 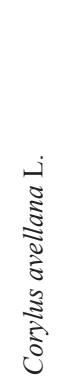 & 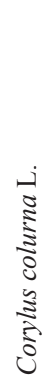 & 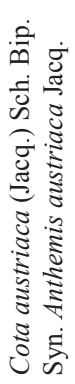 \\
\hline
\end{tabular}




\begin{tabular}{|c|c|c|c|c|c|c|c|c|c|c|}
\hline 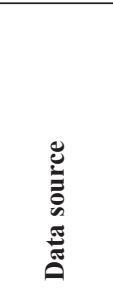 & 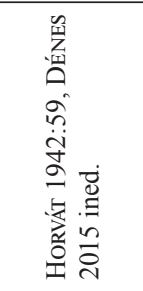 & 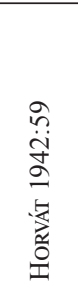 & 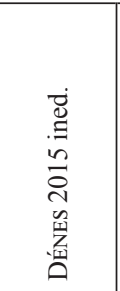 & 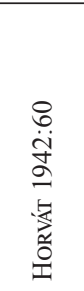 & 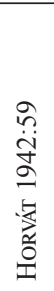 & 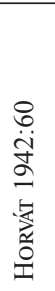 & 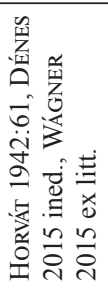 & 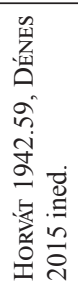 & 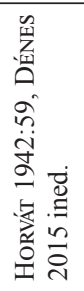 & 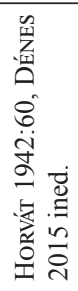 \\
\hline 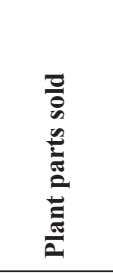 & 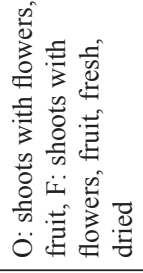 & $\begin{array}{l}0 \\
5 \\
0 \\
0 \\
c \\
c \\
0 \\
5 \\
0 \\
0 \\
0 \\
0\end{array}$ & 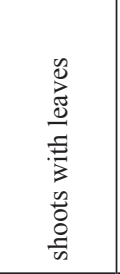 & 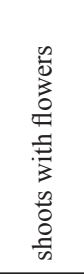 & 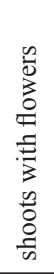 & $\begin{array}{l}n \\
0 \\
0 \\
0 \\
4 \\
0 \\
0 \\
3 \\
0 \\
0 \\
0 \\
\frac{0}{n}\end{array}$ & 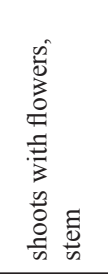 & 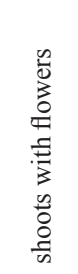 & $\begin{array}{l}n \\
0 \\
0 \\
0 \\
0 \\
0 \\
5 \\
5 \\
0 \\
0 \\
0 \\
0 \\
\frac{0}{n}\end{array}$ & $\stackrel{4}{\tilde{J}}$ \\
\hline ํㅗㄱ & $\sum_{L i}^{0}$ & & 0 & & & & 0 & & & 0 \\
\hline 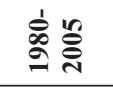 & & & & & & & 0 & ö & $\ddot{\sigma}$ & \\
\hline 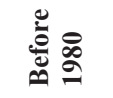 & 0 & 0 & & 0 & 0 & 0 & 0 & 0 & 0 & 0 \\
\hline 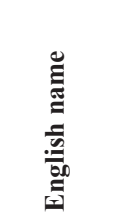 & 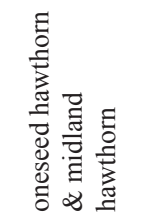 & 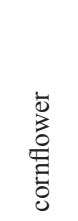 & $\begin{array}{l}\Xi \\
0 \\
0 \\
0 \\
\overline{0} \\
0 \\
0 \\
0\end{array}$ & 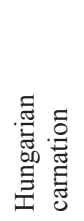 & 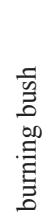 & 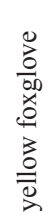 & 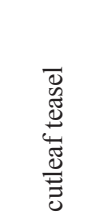 & 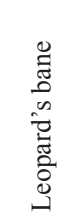 & $\begin{array}{l}\text { 矛 } \\
\text { 总 } \\
\text { 总 } \\
\text { 至 }\end{array}$ & 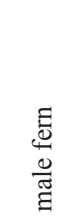 \\
\hline 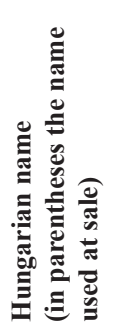 & 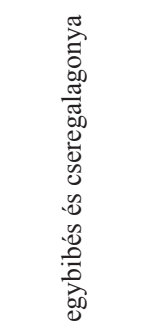 & 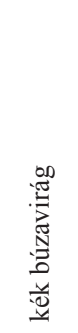 & 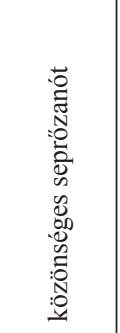 & 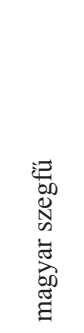 & 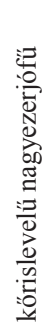 & 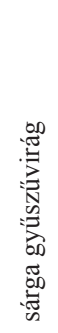 & 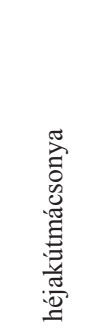 & 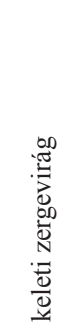 & 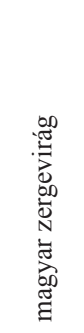 & 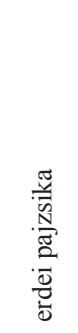 \\
\hline 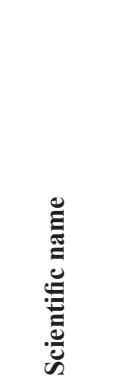 & 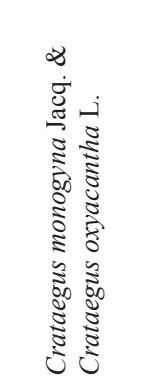 & 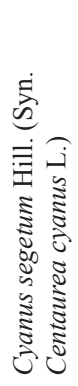 & 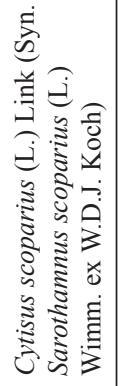 & 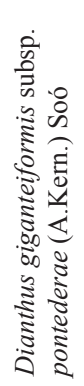 & 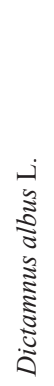 & 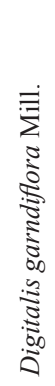 & 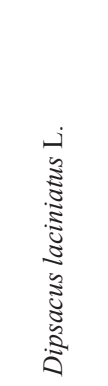 & 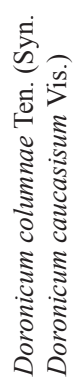 & 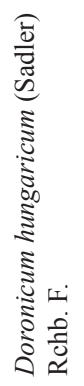 & 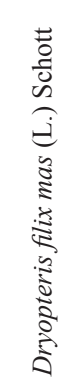 \\
\hline
\end{tabular}


Wild Plants for Sale in the Markets of Pécs Then and Now (Baranya, Hungary) 357

\begin{tabular}{|c|c|c|c|c|c|c|c|c|c|}
\hline 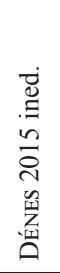 & 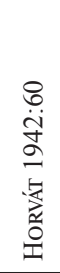 & 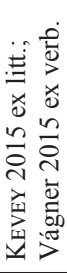 & 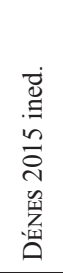 & 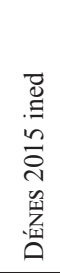 & 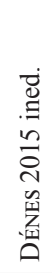 & 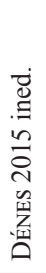 & 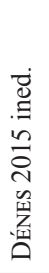 & 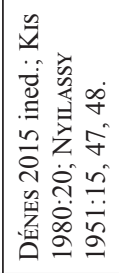 & 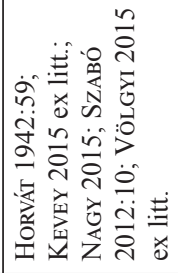 \\
\hline 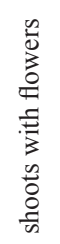 & 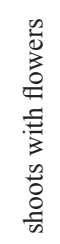 & 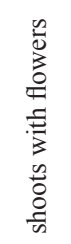 & 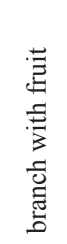 & 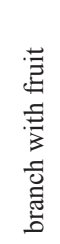 & 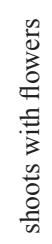 & $\begin{array}{l}\frac{0}{\bar{z}} \\
\overline{0} \\
\text { 咅 }\end{array}$ & 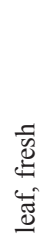 & 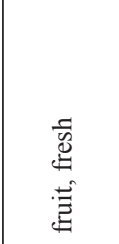 & 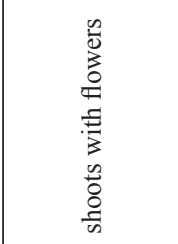 \\
\hline 0 & & & 0 & 0 & 0 & 0 & 古 & 古 & \\
\hline & & 0 & & & & & & & 0 \\
\hline & 0 & & & & & & & I & 0 \\
\hline 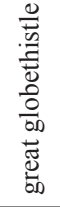 & 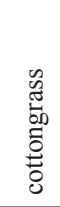 & 恋 & 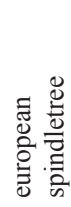 & 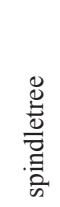 & 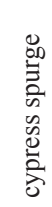 & 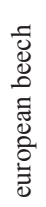 & 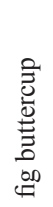 & 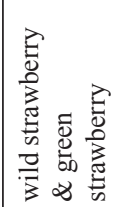 & $\begin{array}{l}\text { वे } \\
\text { t: } \\
0 \\
0 \\
0\end{array}$ \\
\hline 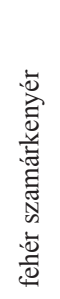 & 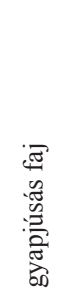 & 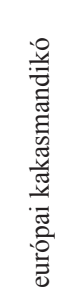 & 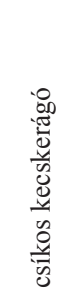 & 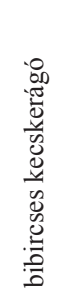 & 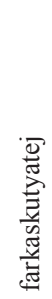 & 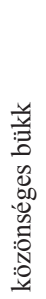 & 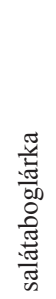 & 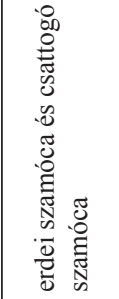 & 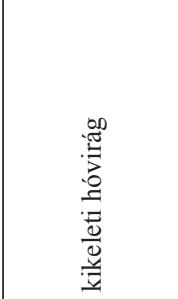 \\
\hline 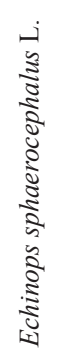 & 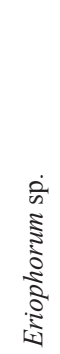 & 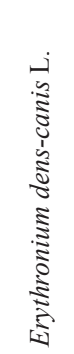 & 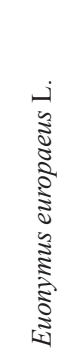 & 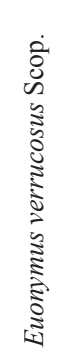 & 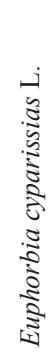 & 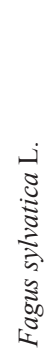 & 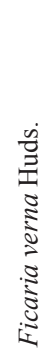 & 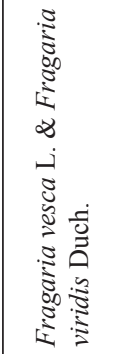 & 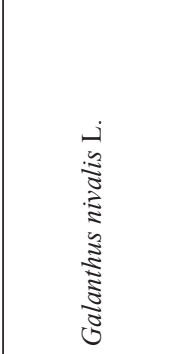 \\
\hline
\end{tabular}




\begin{tabular}{|c|c|c|c|c|c|c|c|c|c|c|}
\hline 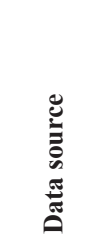 & 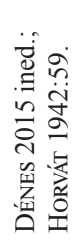 & 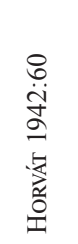 & 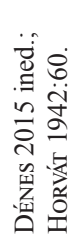 & 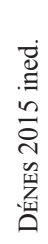 & 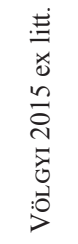 & 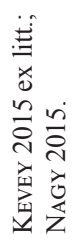 & 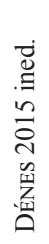 & 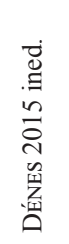 & 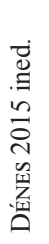 & 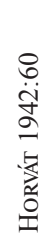 \\
\hline 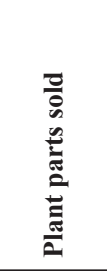 & 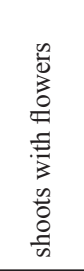 & 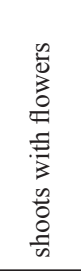 & 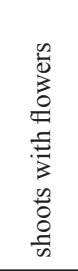 & 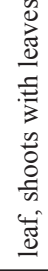 & 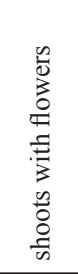 & $\begin{array}{l}n \\
0 \\
0 \\
0 \\
c \\
0 \\
0 \\
3 \\
0 \\
0 \\
0 \\
0 \\
0\end{array}$ & 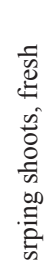 & 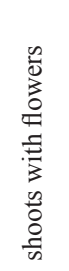 & 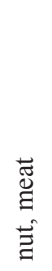 & $\begin{array}{l}n \\
0 \\
0 \\
0 \\
0 \\
0 \\
0 \\
0 \\
0 \\
0 \\
0 \\
0 \\
0\end{array}$ \\
\hline ֻั่ & $\begin{array}{l}\sum^{5} \\
0\end{array}$ & & 0 & 0 & & & 鳬 & 0 & LI & \\
\hline 今ั่ & & & & & 0 & 0 & & & & \\
\hline 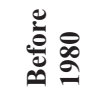 & 0 & 0 & 0 & & & & & & & 0 \\
\hline 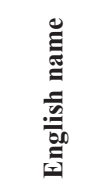 & 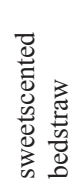 & 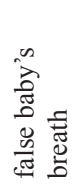 & 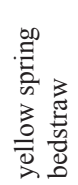 & 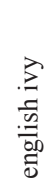 & 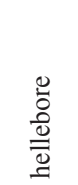 & 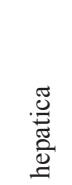 & $\begin{array}{l}\stackrel{0}{0} \\
\text { : } \\
\text { : } \\
\text { : }\end{array}$ & 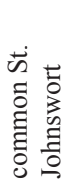 & 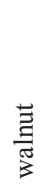 & 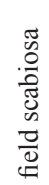 \\
\hline 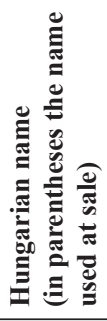 & 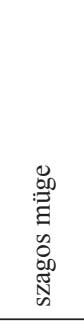 & 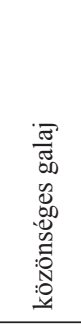 & 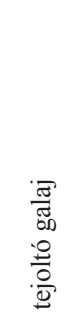 & 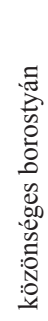 & 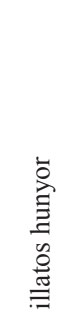 & 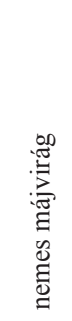 & 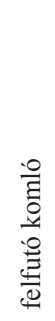 & 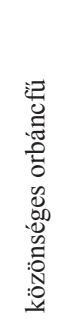 & 㣢 & 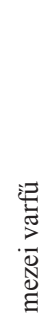 \\
\hline 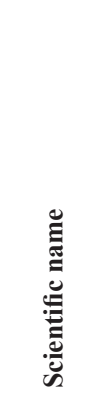 & 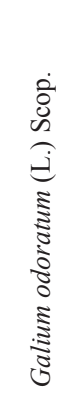 & 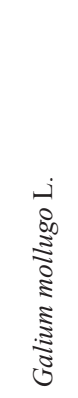 & 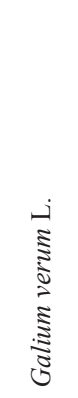 & 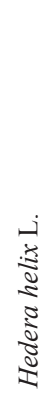 & 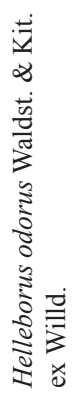 & 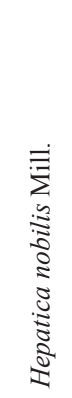 & 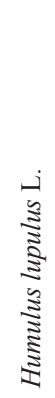 & 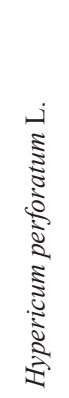 & 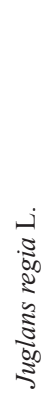 & 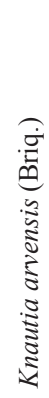 \\
\hline
\end{tabular}




\begin{tabular}{|c|c|c|c|c|c|c|c|c|c|c|}
\hline 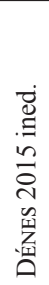 & 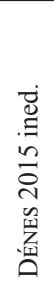 & 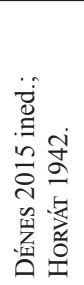 & 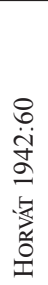 & 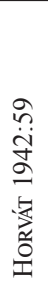 & 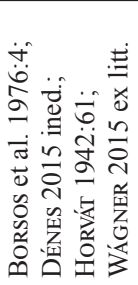 & 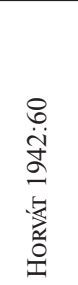 & 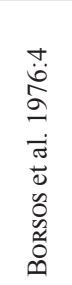 & 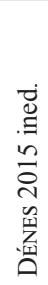 & 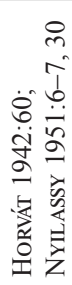 & 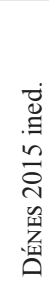 \\
\hline हूँ & 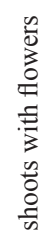 & 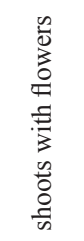 & 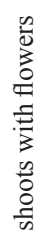 & 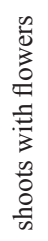 & 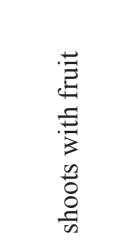 & 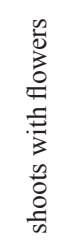 & 墨 & 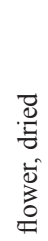 & 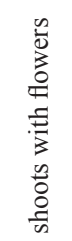 & 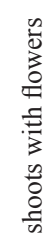 \\
\hline 0 & 0 & 0 & & & 0 & & & 式 & & $\sum_{0}$ \\
\hline & & & & & 0 & & L & & & \\
\hline & & 0 & 0 & 0 & 0 & 0 & & & $\begin{array}{l}0 \\
\Sigma\end{array}$ & \\
\hline 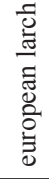 & $\begin{array}{l}\frac{0}{00} \\
\frac{0}{3} \\
\frac{\pi}{3}\end{array}$ & 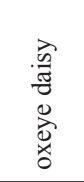 & 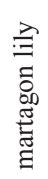 & 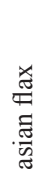 & 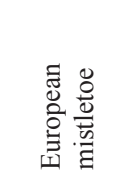 & 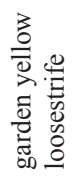 & 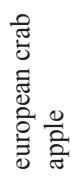 & 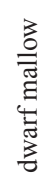 & 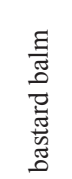 & 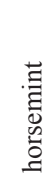 \\
\hline 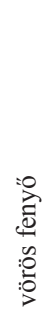 & 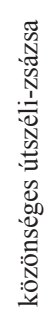 & 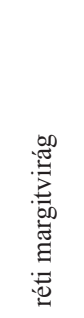 & 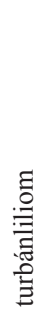 & $\frac{\overline{0}}{\stackrel{0}{0.0}}$ & 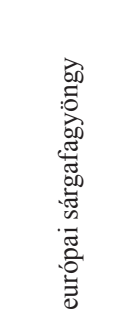 & 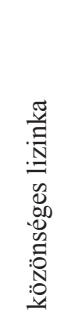 & 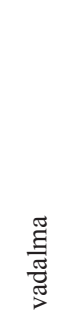 & 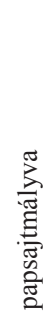 & 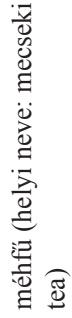 & 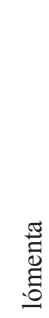 \\
\hline 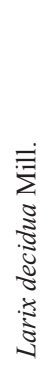 & 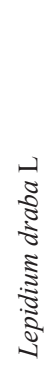 & 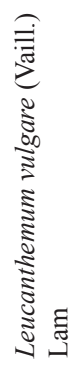 & 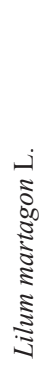 & 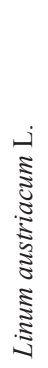 & 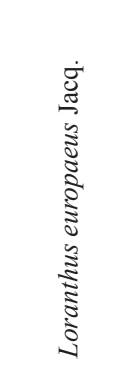 & 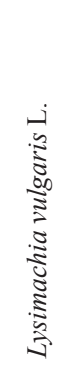 & 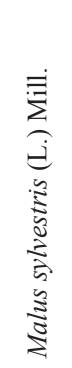 & 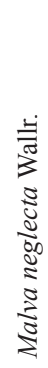 & 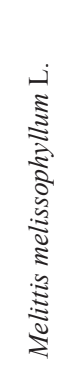 & 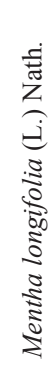 \\
\hline
\end{tabular}




\begin{tabular}{|c|c|c|c|c|c|c|c|c|c|c|}
\hline 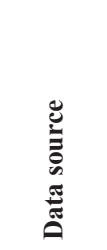 & 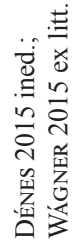 & 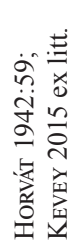 & 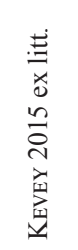 & 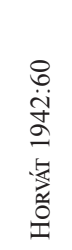 & 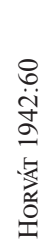 & 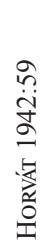 & 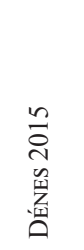 & 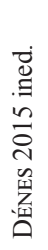 & 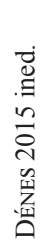 & 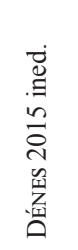 \\
\hline 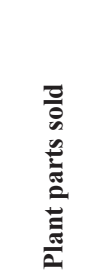 & 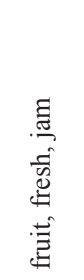 & 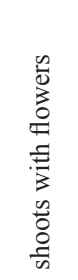 & 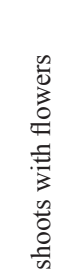 & 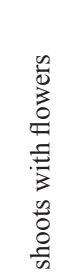 & 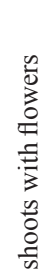 & 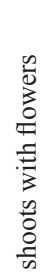 & 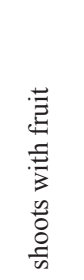 & 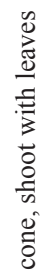 & 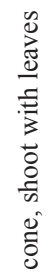 & 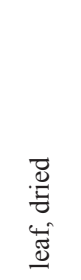 \\
\hline ิㅗ & I & & & & & & 0 & 0 & 0 & $\Sigma$ \\
\hline 今ั่ & L & 0 & 0 & & & & & & & \\
\hline 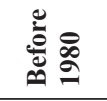 & & 0 & & 0 & 0 & 0 & & & & \\
\hline 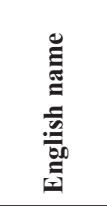 & 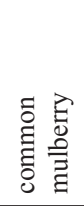 & 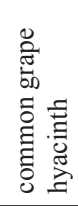 & 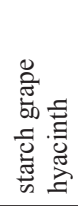 & 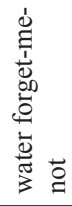 & $\begin{array}{l}\text { 营 } \\
\text { 苞 }\end{array}$ & 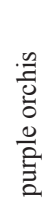 & 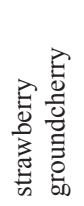 & $\begin{array}{l}\stackrel{.}{0} \\
\frac{\tilde{0}}{0} \\
\frac{\pi}{0}\end{array}$ & $\begin{array}{l}\text { : } \\
0 \\
0 \\
0 \\
0 \\
0\end{array}$ & 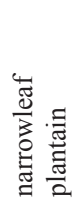 \\
\hline 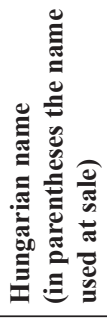 & 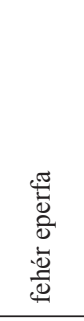 & 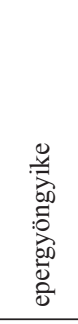 & 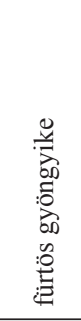 & 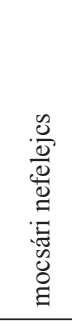 & 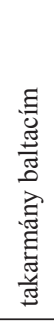 & $\begin{array}{l}\tilde{0} \\
0 \\
0 \\
0 \\
0 \\
0 \\
0 \\
0 \\
0 \\
0\end{array}$ & 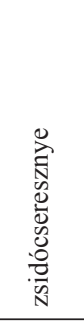 & 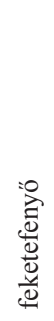 & 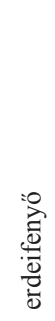 & 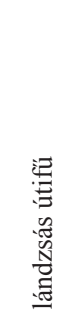 \\
\hline 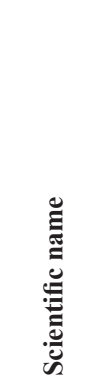 & 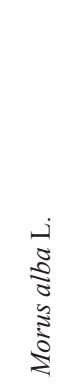 & 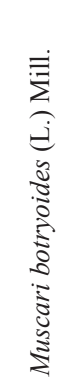 & 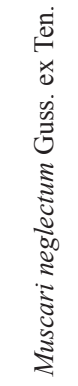 & 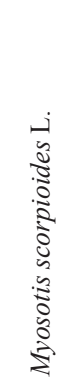 & 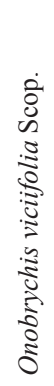 & 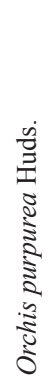 & 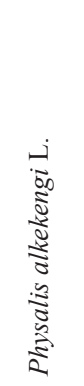 & 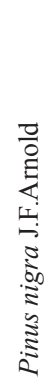 & 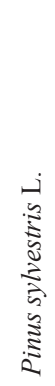 & 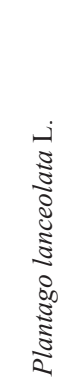 \\
\hline
\end{tabular}


Wild Plants for Sale in the Markets of Pécs Then and Now (Baranya, Hungary) 361

\begin{tabular}{|c|c|c|c|c|c|c|c|c|c|c|}
\hline 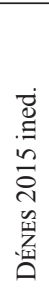 & 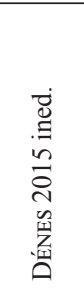 & 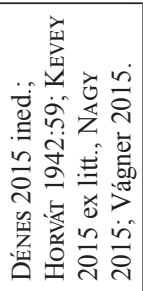 & 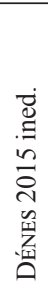 & 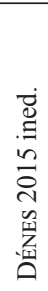 & 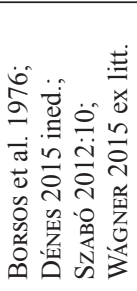 & 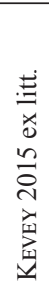 & 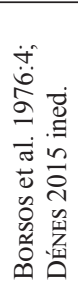 & 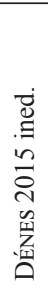 & 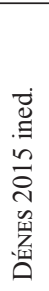 & 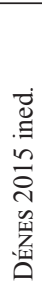 \\
\hline 寻 & 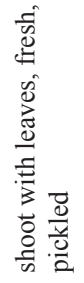 & 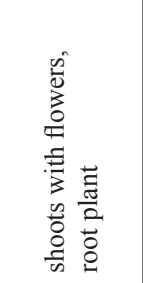 & 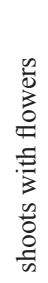 & 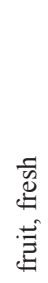 & 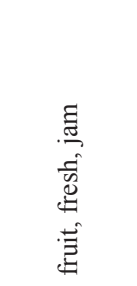 & 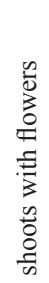 & 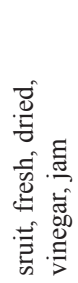 & 声 & 莺 & 墨 \\
\hline 0 & 亲 & ठ̀ & $\check{0}$ & 庄 & I & & 式 & 0 & 0 & 0 \\
\hline & & 0 & & & IL & 0 & & & & \\
\hline & & 0 & & & L & & 工 & & & \\
\hline 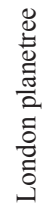 & 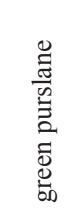 & 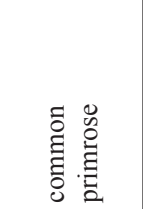 & $\begin{array}{l}\vec{E} \\
\frac{\overrightarrow{0}}{0} \\
\frac{0}{3} \\
\frac{0}{3}\end{array}$ & 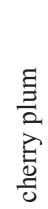 & 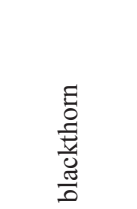 & 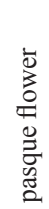 & 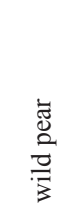 & 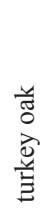 & 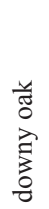 & 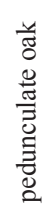 \\
\hline $\begin{array}{l}\frac{\sqrt[G]{\tilde{E}}}{2} \\
\frac{\pi}{2}\end{array}$ & 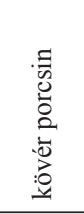 & 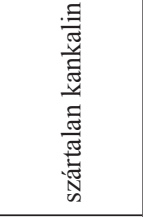 & 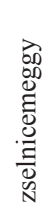 & 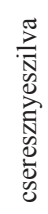 & 离 & 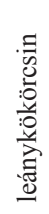 & 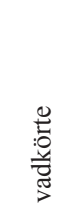 & 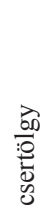 & 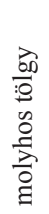 & 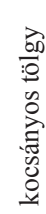 \\
\hline 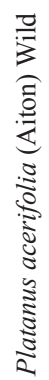 & 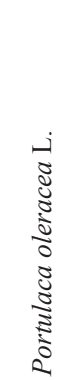 & 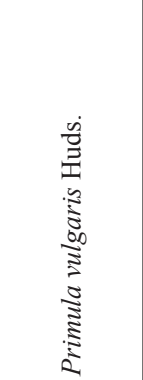 & 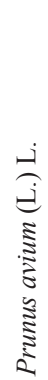 & 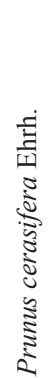 & 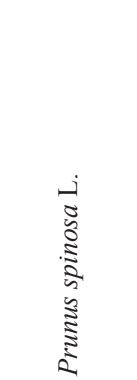 & 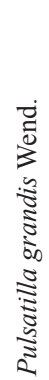 & 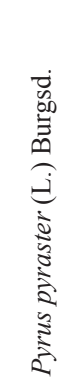 & 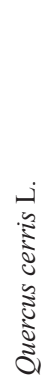 & 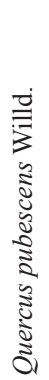 & 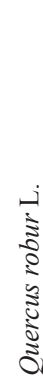 \\
\hline
\end{tabular}




\begin{tabular}{|c|c|c|c|c|c|c|c|c|c|}
\hline 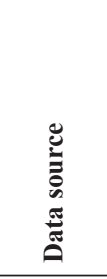 & 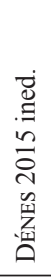 & 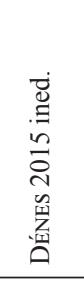 & 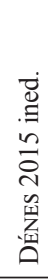 & 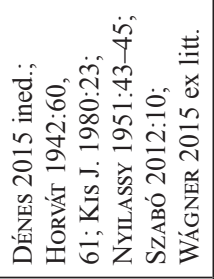 & 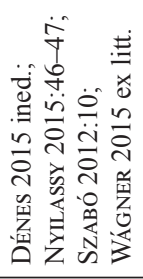 & 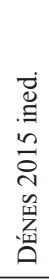 & 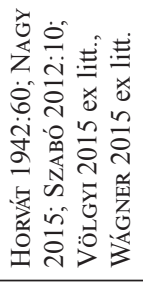 & 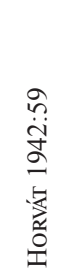 & 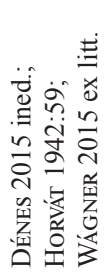 \\
\hline 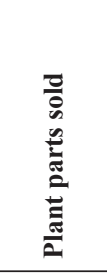 & 壱 & $\begin{array}{l}0 \\
0 \\
0 \\
0 \\
0 \\
0 \\
0 \\
3 \\
0 \\
0 \\
0 \\
0 \\
0\end{array}$ & 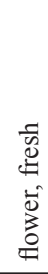 & 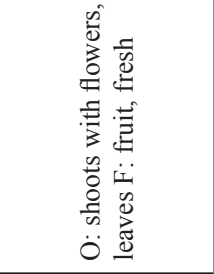 & 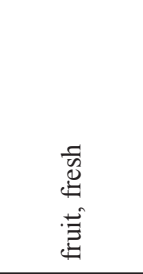 & 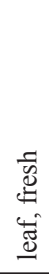 & $\begin{array}{l}\overrightarrow{0} \\
\frac{0}{n}\end{array}$ & $\begin{array}{l}\overrightarrow{0} \\
\frac{8}{n}\end{array}$ & 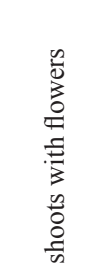 \\
\hline ํㅗㄹ & 0 & 0 & $\sum_{\dot{1}}^{\dot{1}}$ & ${ }_{1}^{0}$ & 工 & 庄 & & & 0 \\
\hline 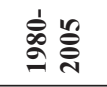 & & & & $0^{\circ}$ & 山 & & 0 & & 0 \\
\hline 竎 。 & & & & ${ }_{1}^{0}$ & L & & 0 & 0 & 0 \\
\hline 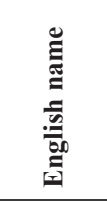 & 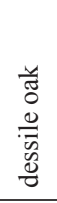 & 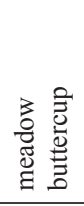 & $\begin{array}{l}\overrightarrow{0} \\
\overrightarrow{0} \\
0 \\
\frac{u}{0} \\
\frac{\pi}{0}\end{array}$ & $\begin{array}{l}0 \\
0 \\
0 \\
0 \\
0 \\
0 \\
0\end{array}$ & $\begin{array}{l}\vec{E} \\
\frac{0}{0} \\
\frac{0}{0} \\
\frac{\pi}{0}\end{array}$ & $\begin{array}{l}\bar{\Xi} \\
\bar{\Xi} \\
0\end{array}$ & $\begin{array}{l}\Xi \\
0 \\
0 \\
0 \\
0 \\
\tilde{0} \\
\overline{0} \\
0 \\
0\end{array}$ & 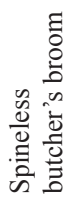 & $\begin{array}{l}\frac{z}{0} \\
0 \\
\frac{7}{3} \\
0 \\
0 \\
0 \\
0 \\
0\end{array}$ \\
\hline 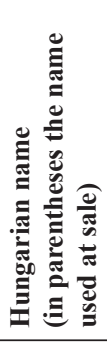 & 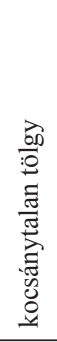 & 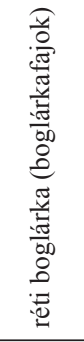 & 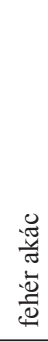 & 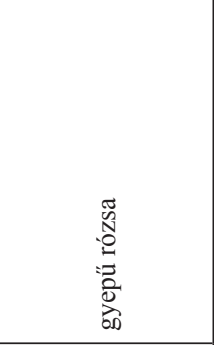 & 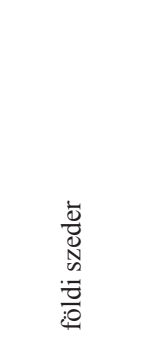 & 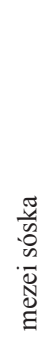 & $\begin{array}{l}0 \\
0.0 \\
0 \\
0 \\
\frac{0}{0} \\
0 \\
0 \\
0 \\
0 \\
0 \\
0 \\
0 \\
\infty\end{array}$ & 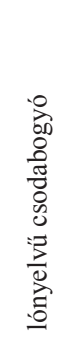 & 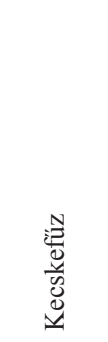 \\
\hline 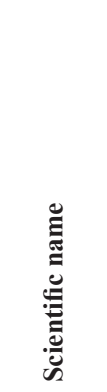 & 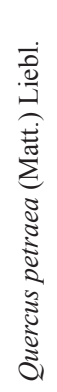 & 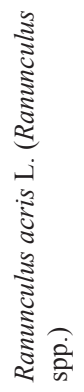 & 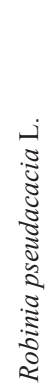 & 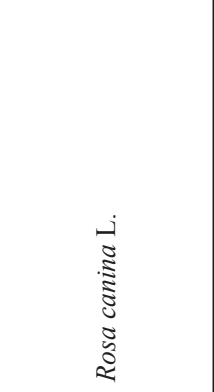 & 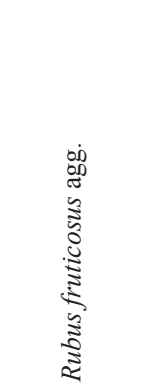 & 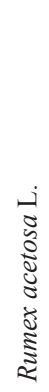 & 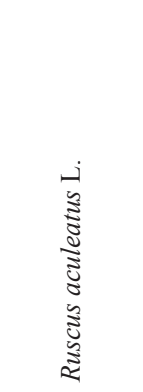 & 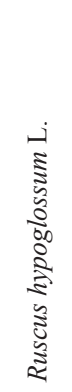 & 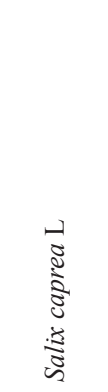 \\
\hline
\end{tabular}


Wild Plants for Sale in the Markets of Pécs Then and Now (Baranya, Hungary) 363

\begin{tabular}{|c|c|c|c|c|c|c|c|c|c|c|}
\hline 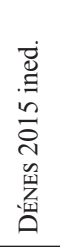 & 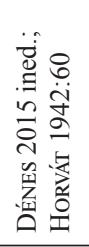 & 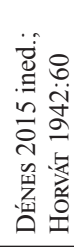 & 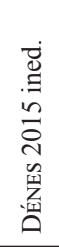 & 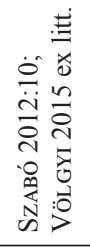 & 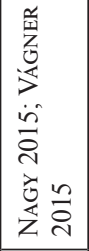 & 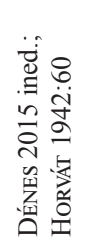 & 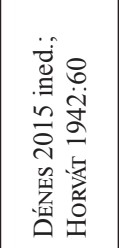 & 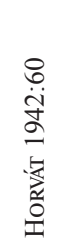 & 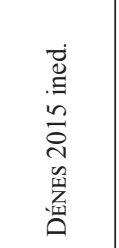 & 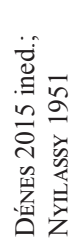 \\
\hline 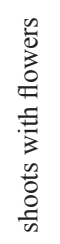 & 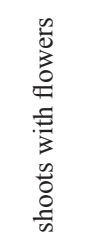 & 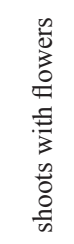 & 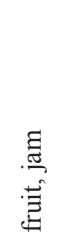 & 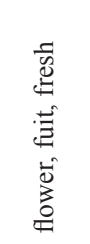 & 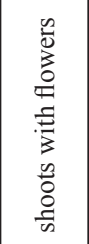 & 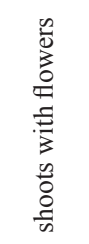 & 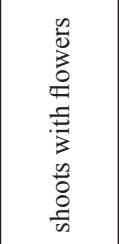 & 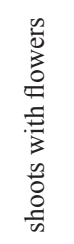 & 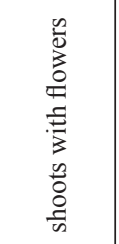 & 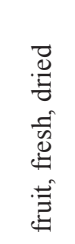 \\
\hline 0 & 0 & 0 & 占 & L & & 0 & ö & & 0 & 它 \\
\hline & 0 & 0 & & & & 0 & 0 & 0 & & I \\
\hline 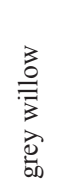 & 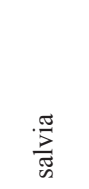 & 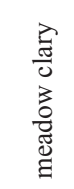 & 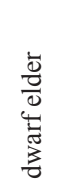 & 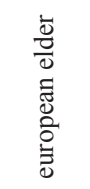 & 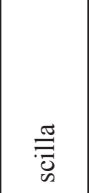 & 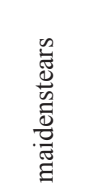 & 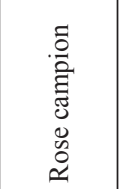 & 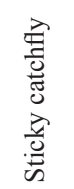 & 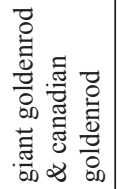 & 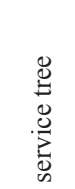 \\
\hline 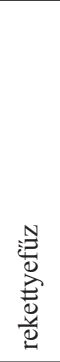 & 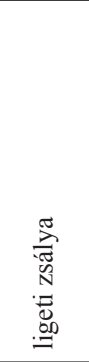 & 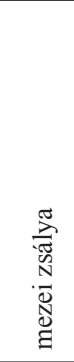 & $\begin{array}{l}\frac{\pi}{8} \\
\text { : } \\
: 0 \\
0 \\
0\end{array}$ & 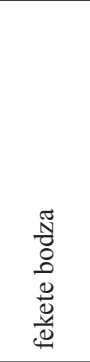 & 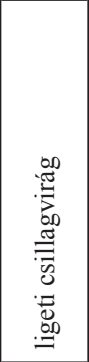 & 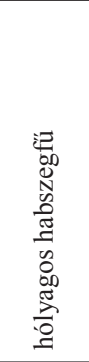 & 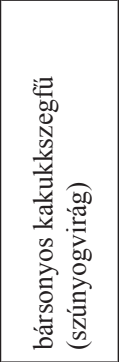 & 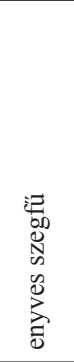 & 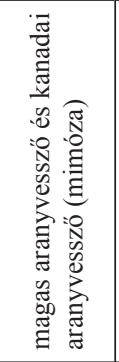 & 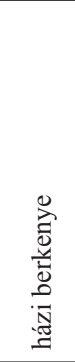 \\
\hline 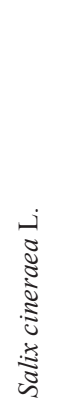 & 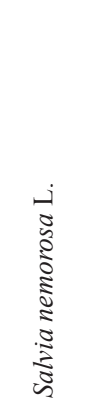 & 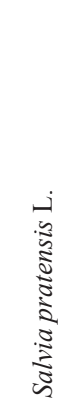 & 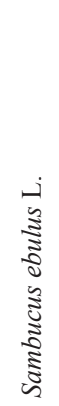 & 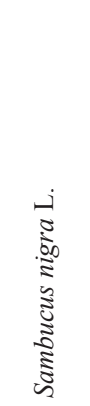 & 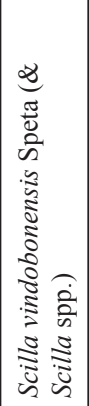 & 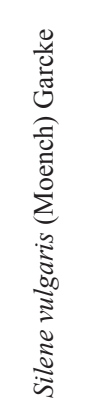 & 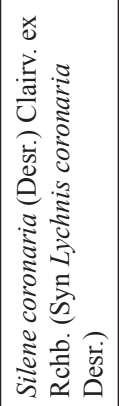 & 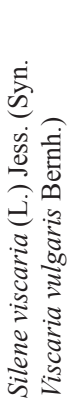 & 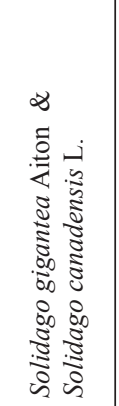 & 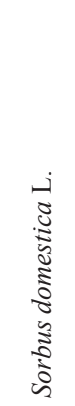 \\
\hline
\end{tabular}




\begin{tabular}{|c|c|c|c|c|c|c|c|c|c|}
\hline 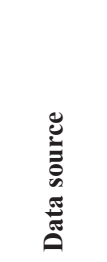 & 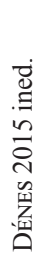 & 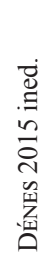 & 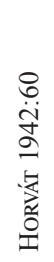 & 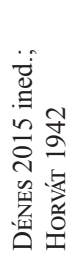 & 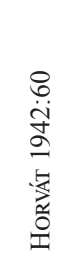 & 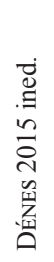 & 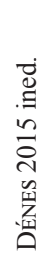 & 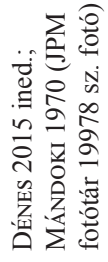 & 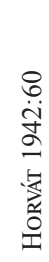 \\
\hline 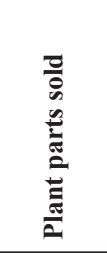 & 莺 & 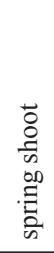 & $\begin{array}{l}\text { n } \\
0 \\
0 \\
0 \\
4 \\
0 \\
3 \\
0 \\
0 \\
0 \\
\frac{0}{n}\end{array}$ & 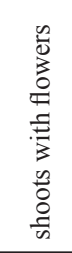 & 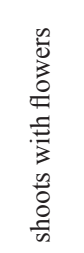 & 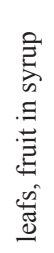 & $\begin{array}{l}n \\
0 \\
0 \\
0 \\
c \\
0 \\
0 \\
0 \\
0 \\
0 \\
0 \\
\frac{0}{n}\end{array}$ & $\begin{array}{l}\overrightarrow{0} \\
\text { 言 }\end{array}$ & $\begin{array}{l}n \\
0 \\
0 \\
0 \\
0 \\
0 \\
0 \\
0 \\
0 \\
0 \\
0 \\
\frac{0}{n}\end{array}$ \\
\hline ֻั่ & 0 & L & & & & LI & $\sum_{L=}$ & $\Sigma$ & \\
\hline 今ั่ & & & & 0 & & & & & \\
\hline 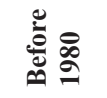 & & & 0 & 0 & 0 & & & $\Sigma$ & 0 \\
\hline 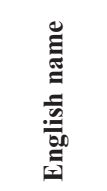 & $\begin{array}{l}\vec{\Xi} \\
\overline{0} \\
\frac{\overrightarrow{0}}{0} \\
\frac{\pi}{0}\end{array}$ & 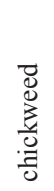 & 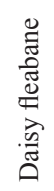 & 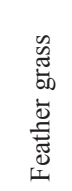 & 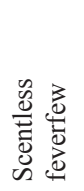 & 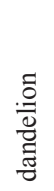 & $\underset{\Xi}{\rightleftarrows}$ & 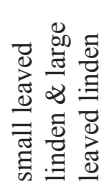 & $\begin{array}{l}\overline{0} \\
\frac{0}{0}\end{array}$ \\
\hline 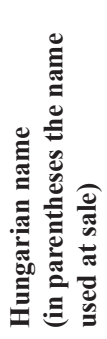 & 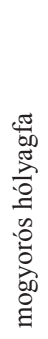 & 兽 & 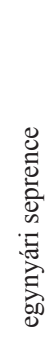 & 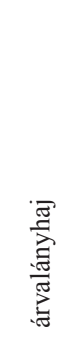 & 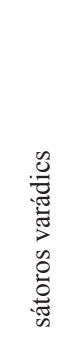 & 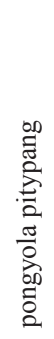 & 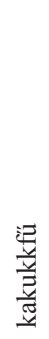 & 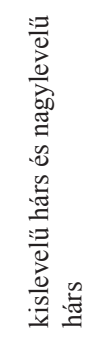 & 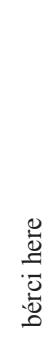 \\
\hline 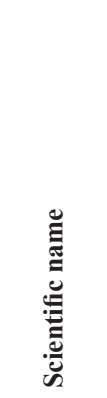 & 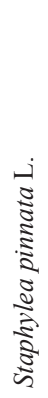 & 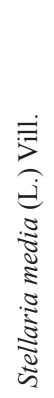 & 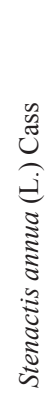 & 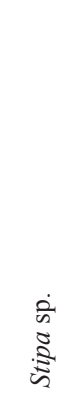 & 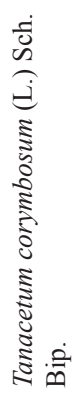 & 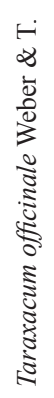 & 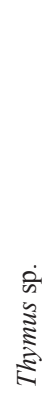 & 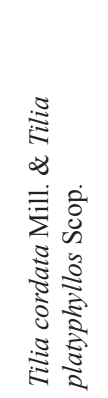 & 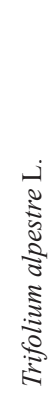 \\
\hline
\end{tabular}




\begin{tabular}{|c|c|c|c|c|c|c|c|}
\hline 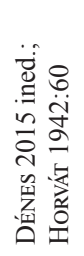 & 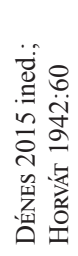 & 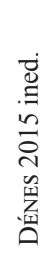 & 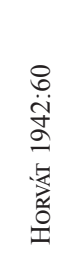 & 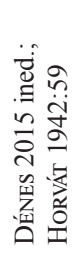 & 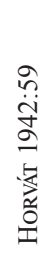 & 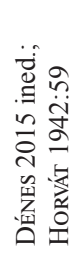 & 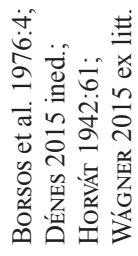 \\
\hline 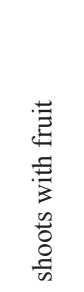 & 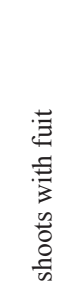 & 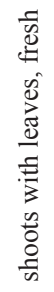 & 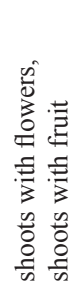 & 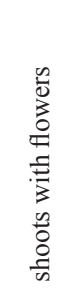 & $\begin{array}{l}n \\
0 \\
0 \\
0 \\
0 \\
0 \\
0 \\
0 \\
n \\
0 \\
0 \\
0 \\
n\end{array}$ & $\begin{array}{l}n \\
0 \\
0 \\
0 \\
0 \\
0 \\
0 \\
0 \\
0 \\
0 \\
0 \\
0 \\
0\end{array}$ & 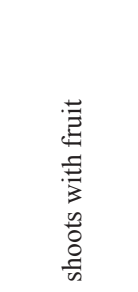 \\
\hline 0 & 0 & $\sum_{L=}^{H}$ & 0 & 0 & & 0 & 0 \\
\hline 0 & 0 & & 0 & 0 & 0 & 0 & 0 \\
\hline 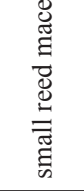 & 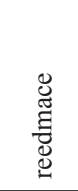 & 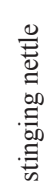 & 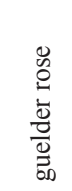 & $\frac{\overrightarrow{\mathrm{d}}}{\frac{\mathrm{O}}{\mathrm{T}}}$ & $\frac{\overrightarrow{0}}{\frac{0}{7}}$ & 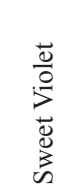 & 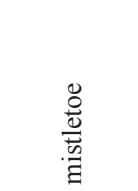 \\
\hline 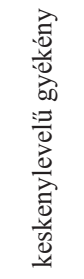 & 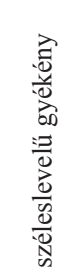 & 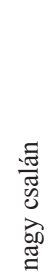 & 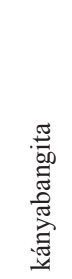 & 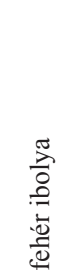 & 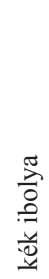 & 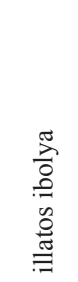 & 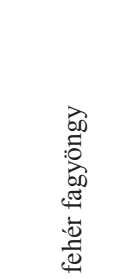 \\
\hline 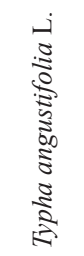 & 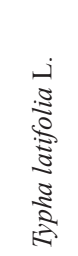 & 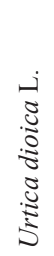 & 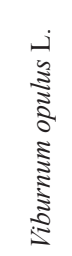 & 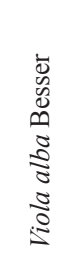 & 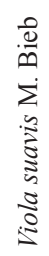 & 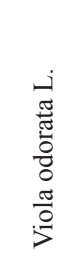 & 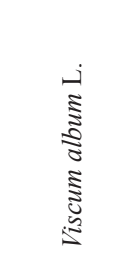 \\
\hline
\end{tabular}




\section{REFERENCES CITED}

ANDRÁSFALVY, Bertalan

1965 A sárköziek gazdálkodása a XVIII. és XIX. században. [Agriculture in Sárköz during the $18^{\text {th }}$ and $19^{\text {th }}$ century]. Dunántúli Dolgozatok 3:16.

ARADI, Csaba - DÉvAI, György - FinTHA, István - HorváTH, Klára - BANCSI, István - B. TóтH, Mária - ÖTvös, János

1975 Tanulmányok Haláp élővilágáról [Studies about Haláp’s Wildlife] [Studien über Haláps Lebenswelt] Debrecen Déri Múzeum Évkönyve 1974:13-156.

BARINA, Zoltán

2014 A medvehagyma (Allium ursinum). [Wild Garlic (Allium ursinum)]. Magyar Természettudományi Múzeum Blog http://mttmuzeum.blog.hu/2014/04/03/a medvehagyma_allium_ursinum, (accessed January 9, 2016.)

BARTHA, Gabó

2015 Húsvét Nagybányán. [Easter in Baia Mare [Nagybánya]]. http://slowfood. Boros, Ádám blog.hu/2015/05/10/husvet_nagybanyan (accessed February 22, 2016.)

1924 A budapesti virágkereskedelem vadvirágai. [Wild Flowers in the Budapest Flower Trade] Természettudományi Közlöny 56:382-383.

1968 Virágszedés és természetvédelem. [Flower Picking and Nature Conservancy.] Búvár 13(3):148-149.

1947 A pesti utca vadvirágai. [Wild Flowers in the Streets of Pest.] Budapest $3: 153-158$.

Borsos, Sándor - Dobos, László - CSERMAVÖLGYI, Imre

1976 Erdők élete Magyaregregyen. Magyaregregyi erdők és erdőbirtokosság. Néprajzi pályázat. A Magyaregregyi Honismereti Szakkör, vezető: Csermavölgyi Endréné. [The Life of Forests in Magyaregregy. Forests and Forest Ownership in Magyaregregy.] Ethnographic Contest. The Magyaregregy Homeland Study Group, Head: Csermavölgyi Endréné. Manuscript. Janus Pannonius Múzeum Néprajzi Osztály adattára, Lsz.: 38-76.

DANKó, Imre

1965 Adalékok a pécsi piacok és vásárok néprajzához. [Supplements to the Ethnography of Markets and Fairs in Pécs]. Janus Pannonius Múzeum Évkönyve 1964:169-185.

DÉnes, Andrea - PAPP, Nóra - BABAI, Dániel - CzúCz, Bálint - MolnáR, Zsolt

2012 Wild Plants Used for Food by Hungarian Ethnic Groups Living in the Carpathian Basin. Acta Soc Bot Pol. 81(4): 381-396. http://dx.doi.org/10.5586/ asbp.2012.040, (accessed December 20, 2015.)

DÉNes, Tünde - TóTH, Mónika - GYERGYÁK, Kinga - LöRINCZ, Péter-VARGA, Erzsébet PAPP, Nóra

2014 Szemelvények Homoródalmás (Erdély) népi gyógynövényismeretéből [Excerpts from the Vernaculal Knowledge of Herbs in Homoródalmás (Transylvania)] Botanikai Közlemények 101(1-2):227-241. 
Dogan, Yunus - Ugulu Ilker - DuRKan Nazmi

2013 Wild Edible Plants Sold in the Local Markets of Izmir, Turkey. Pak. J. Bot., 45(S1): 177-184. http:/www.researchgate.net/publication/286322823 (accessed December 20, 2015.)

Dogan, Yunus - Nedelcheva, Anely

2015 Wild Plants from Open Markets on Both Sides of the Bulgarian-Turkish Border. Indian Journal of Traditional Knowledge 14(3):351-358. http://www. researchgate.net/publication/280803105 (accessed December 20, 2015.)

FeHÉr, Orsolya - VArga, Adrienne

2013 A medvehagyma termesztése és felhasználása. [The Cultivation and Use of Wild Garlic.] Biokultúra 2013/1. http://www.biokontroll.hu/cms/index. php?option $=$ com_content $\&$ view $=$ article $\&$ id $=1553$ :a-medvehagymatermesztese-es-felhasznalasa\&catid=115:gyujtottnovenyekbiodrogokfitoterap ia\&Itemid=43\&lang=hu (accessed January 25, 2016.)

FüVESY, Anikó

1997 Gyüjtögetés és erdőélés Mozsgó környékén. [Foraging and Forestry around Mozsgó.] Tiscium 10:207-217.

Grynaeus, Tamás

1964 Gyógynövényárusok Szeged piacain. [Vendors of Herbs at the Markets of Szeged.] In Palla, Ákos (ed) Az Országos Orvostörténeti Könyvtár Közleményei [Publications of the National Medical History Library] 30:89126. Budapest: Medicina Egészségügyi Könyvkiadó.

GundA, Béla

1956 Az Ormánság múltjáról. [On the History of the Ormánság]. In Gunda, Béla (ed) Néprajzi gyüjtöúton [On an Ethnographic Field Trip], 17-55. Debrecen: Alföldi Magvető Kiadó.

1977 Baraboly [Chervil]. In ORTUTAY, Gyula (ed. in chief) Magyar Néprajzi Lexikon [Hungarian Ethnographic Lexicon] 1:216.

1981 Sulyom [Water Chestnut]. In OrTUTAY, Gyula (ed. in chief) Magyar Néprajzi Lexikon [Hungarian Ethnographic Lexicon] 4:499.

2001 A vadnövények gyüjtése. [Foraging for Wild Plants]. In PALÁDI-KovÁcs, Attila (ed in chief) Magyar Néprajz II. Gazdálkodás [Hungarian Ethnography II. Economy], 11-40. Budapest: Akadémiai Kiadó.

Horvát, Adolf Olivér

1940 A pécsi piac vadvirágai [Wild Flowers in the Markets of Pécs]. Búvár 1940(6): 257-259.

1942 Adatok a növényzetünk és a nép kapcsolataihoz. A pécsi piac vadvirágai. [Data on the Relationship between Plants and People. Wild Flowers in the Markets of Pécs]. In Honvát, Adolf Olivér (ed) A Mecsekhegység és déli síkjának növényzete [The Vegetation of the Mecsek Mountains and its Southern Plains], 59-61. Pécs: A Ciszterci Rend Kiadása. 
KEREKI, Albert

2001 Egy rózsaszál szebben beszél... A vadvirág-kereskedelem pusztító következményeiről. A hagyd élni a vadvirágot WWF kampány propagandafüzete. [A Rose Speaks Better.... On the Devastating Consequences of the Wildflower Trade. Campaign booklet of the WWF, Let the wildflowers live.] WWF Hungary, Budapest. http://www.wwf.hu/media/file/1185721528_ Egy_rozsaszal_szebben_beszel.pdf (accessed December 15, 2015.)

KIRÁLY, István

2001 Bolgárkertészet Pécsett (Irodalmi áttekintés). [Bulgarian Gardening in Pécs. (Literature Review)]. Acta Agraria Kaposváriensis 5(2): 83-90.

KIss, Jozsef

1980 Paraszti ételek a század első felében a Szigetvár környéki falvakban (Botyka, Nemeske) [Peasant Food in the First Half of the Century in Villages around Szigetvár (Botyka, Nemeske)]. Janus Pannonius Museum, Ethnographic Repository, Inventory number 255/1078. 21.

KIss, Lajos

1929 Nyírvíz [Birch Water]. A Földgömb 1(1):1-6.

Kiss Z., Géza

1994 Zsákmányoló foglalkozások: Halászat, gyüjtögetés [Predatory Occupations: Fishing, Gathering]. In A régi Vajszló 1244-1849 [The old Vajszló 1244-1849]. 174-179. Pécs: Vajszló Nagyközség Önkormányzata.

ŁucZaJ, Łukasz - Zovko-Končić, Marijana - MitıčEvić, Tihomir - Dolina, Katija PANDŽA, Marija

2013 Wild Vegetable Mixes Sold in the Markets of Dalmatia (Southern Croatia). Journal of Ethnobiology and Ethnomedicine 9:2. https://ethnobiomed. biomedcentral.com/articles/10.1186/1746-4269-9-2 (accessed January 4, 2016.)

ŁucZaj, Łukasz - Pieroni, Andrea - TARdío, Javier - PARdo-De-SANTAYANa, Manuel SõukAND, Renata - Svanberg, Ingvar - Kalle, Raivo

2012 Wild Food Plant Use in $21^{\text {st }}$ Century Europe: The Disappearance of Old Traditions and the Search for New Cuisines Involving Wild Edibles. Acta Societatis Botanicorum Poloniae 81(4):359-370. http://dx.doi.org/10.5586/ asbp.2012.03 (accessed January 4, 2016.)

MÁTHÉ, Imre

1938 A debreceni virágpiac vadvirágai és azok nyírségi és tiszántúli elterjedése [Wild Flowers in Debrecen's Flower Markets and Their Diffusion in the Nyírség and Transtisza Regions]. Tiszántúli Kertészeti Tanácsadó. 1(2):2.

MÉszÁRos B., Endre

2015 Kinccsé vált a helyi érték [Local Valuables Turned into Treasure]. Új Dunántúli Napló. 2015(április 11.):12.

Molnár V., Attila

2014 Természetvédelmi botanika oktatási segédanyag a debreceni egyetem kurzusához. [Environmental Conservation Botany Educational Course Aids at Debrecen University.] Debrecen, 64. https://www.researchgate.net/ publication/268955908_Termeszetvedelmi_botanika_Oktatasi_segedanyag_a Debreceni_Egyetem_kurzusahoz (accessed December 15, 2015.) 
Müller, Géza

1973 Gyűjtögető életmód szőkédi hagyományai [Traditions of a Foraging Lifestyle

NAGY, Jenő in Szökéd] Baranyai Müvelödés 3:156-157.

1917 A Magyar Alföld két pusztuló növénye a kolokán és a sulyom [Water Pineapple and Water Chestnut: Two Vanishing Plants on the Great Hungarian Plain]. Természettudományi Közlöny 49:838-839.

NaGY, Rózsa

1942 Adatok a Baranya megyei Nagyváty növényekkel kapcsolatos szokásaihoz és néphagyományaihoz [Data on the Customs and Folk Traditions Regarding Plants in Nagyváty, Baranya County]. Magyar Népnyelv 4:268-308. http:// www.adatbank.ro/html/alcim_pdf9855.pdf (accessed December 21, 2015.)

NÉMETH, Ferenc - SEREGÉLYES, Tibor

1982 Ne bántsd a virágot. Néhány ritkaság a hazai növényvilágból [Do Not Harm the Flowers. Some Rarities of Our Domestic Flora]. 132. Budapest: Országos Környezet- és Természetvédelmi Hivatal.

NyILASSY, Judit

1951 Táplákozás Zengővárkonyon. Janus Pannonius Múzeum, Néprajzi adattár (Lsz.: 7-75). Cédulajegyzék. [Nutrition in Zengővárkony. Janus Pannonius Museum, Ethnographic Repository (No.: 7-75). Index tag.] 232.

PAPP, Nóra - HorváTH, Dávid

2013 Vadon termő ehető növények Homordókarácsonyfalván [Wild Growing Edible Plants in Homoródkarácsonyfalva]. Dunántúli Dolgozatok (A) Természettudományi sorozat 13:83-92.

PÉNZES, Antal

1926a Budapesti virágok [Flowers in Budapest]. Kertészeti Lapok 8:113-114

1926b Budapesti virágok [Flowers in Budapest]. Kertészeti Lapok 9:130-131.

Petrikovits, László

1943 A sulyomtermés gyüjtése és felhasználása a Tisza vidékén [The Gathering and Use of Water Chestnut in the Tisza Region]. Természettudományi Közlöny $75: 216$.

RAPAICS, Raymund

1932 A magyarság virágai: a virágkultusz története. [The Flowers of the Hungarians: History of the Flower Cult]. 179-180. Budapest: Természettudományi Társulat. http://www.tankonyvtar.hu/hu/tartalom/tkt/magyarsag-viragai/ch13. html (accessed December 19, 2015.)

SCHNEIDER, Ede

1976 Röviden [Briefly]. A Hét 21/14:11.

SzaBó, László Gy.

2012 Az utolsó bissei virágszedő asszony [The Last Flower Picking Woman in Bisse]. Farmakognóziai Hírek 7(23):10.

Temesi, Géza

2014 Nagy hasznot hoz a medvehagyma lopása [Theft of Wild Garlic Brings Great Benefits.] Országos Természtör Egyesület Hírek 2014/04. http://www. termeszetor.hu/2014/04/, (accessed January 25, 2016.) 
TÉTÉNYI, Péter

1995 A Gyógynövény Kutató Intézet története (1915-1990) 1. rész [History of the Medicinal Plant Research Institute (1915-1990) Part 1]. Gyógyszerészet 39:503-509.

Timár, Lajos

1953 Vadvirágok Szeged piacain [Wild Flowers in the Markets of Szeged]. Agrártudományi Egyetem Kert- és Szőlőgazdaságtudományi Kar Évkönyve 1951(2):109-116.

Új Szó

$197326(35): 8$.

ÚJVÁRI, Zoltán

1957 A vadontermő növények szerepe a táplálkozásban az abaúj-zempléni hegyvidéken [The Role of Wild Plants in the Diet of the Mountains of AbaújZemplén]. Néprajzi Értesitö 39:231-244.

VÁRÓCZI, Zsuzsa

2011 Vadon élő növények a bukovinai székelyek táplálkozásában [Wild Edible Plants in the Diet of the Bukovina Szeklers / Székelys (the Hungarians)]. In Ehető vadnövények a Kárpát-medencében [Edible Wild Plants in the Carpathian Basin]. Dunántúli Dolgozatok (A) Természettudományi Sorozat. [Studia Pannonica (A) Series Historico Naturalis] 13:13-20. Pécs: Janus Pannonius Múzeum.

VesZELSZKi A.

1798 A növényplánták országából való erdei és mezei gyüjtemény, vagy-is fa- és füszeres könyv. [A Collection of Forest and Field Plants, or The Book of Trees and Herbs]. Kiadó nélkül. Pesthen.

VIGA, Gyula

1986 Tevékenységi formák és javak cseréje a Bükk-vidék népi kultúrájában [Activity Forms and Exchange of Goods in the Folk Culture of the Bükk Region] Borsodi Kismonográfiák 23. Miskolc.

1990 Árucsere és migráció Észak-Magyarországon [Exchange of Goods and Migration in Northern Hungary]. Miskolc.

WIRTH, Tamás - KovÁCS, Dániel- DÉNES, Andrea- CsIKY, János

2010 Elszigetelődött diverzitási centrumok Pécsett I.: a Havi-hegy flórája száznyolcvan év tükrében [Isolated Diversity Centers in Pécs I.: Our Knowledge of the Flora of Havi Hill, Changes over the Last 180 Years]. In DÉNES, Andrea (ed) Pécs és környéke növényvilága egykor és ma. Botanikai és tájtörténeti tanulmányok [The Vegetation of Pécs and Its Surroundings, Past and Present. Studies of the Botany and the Landscape History] Dunántúli Dolgozatok (A) Természettudományi Sorozat. [Studia Pannonica (A) Series Historico Naturalis] 12:61-78. Pécs: Baranya Megyei Múzeumok Igazgatósága.

ZENTAI, János

1966 Egy letünt életforma képviselője az Ormánságban [Letzter Vertreter einer untergegangenen Lebensform in der Ormánság] [The Last Representative of a Lost Life Form in the Ormánság]. A Janus Pannonius Múzeum Évkönyve 1965:181-204. 
ZOLTAI, Lajos

1902 Debreczen 200 év előtt. Gazdaságtörténelmi adatok. [Debreczen 200 Years Ago. Data of Economic History.] Magyar Gazdaságtörténelmi Szemle 9:1-29.

Andrea Dénes is a research scientist at the Janus Pannonius Museum of Natural Sciences in Pécs, where she is a botanist and chief museologist. She has been conducting research on flora, plant associations, landscape history and nature conservation in Baranya County, the Villány Mountains and the Drava Plateau since 1989. A few years ago, she also undertook ethnobotanical research on the topic of wild plant use in Hungary, with a particular emphasis on wild plants used as food and their processing. 
